\title{
Excess winter mortality in England and Wales, 2008/09 (provisional) and 2007/08 (final)
}

Vanessa Fearn and Jane Carter office for National Statistics

\section{Key findings}

- There were an estimated 36,700 excess winter deaths in England and Wales in 2008/09. This is an increase of 49 per cent compared with figures for 2007/08.

- The estimate of excess winter deaths in 2008/09 is the highest since 1999/2000.

- In 2008/09 there were 15,300 excess winter deaths in males and 21,400 excess winter deaths in females. The majority of these deaths occurred among those aged 75 and over.

\section{Introduction}

This report analyses provisional estimated figures for excess winter mortality (EWM) for the winter period 2008/09, and final figures for the winter period 2007/08 for deaths occurring in England and Wales. Historical trends in EWM are presented for people in England and Wales from 1950/51 to 2008/09. Figures by sex, age, and Government Office Region of England, and Wales are presented for the five-year period 2004/05 to 2008/09, and by cause of death from 2005/06 to 2007/08. Monthly mortality data for deaths occurring in 2007 and 2008 are presented in the Appendices.

England and Wales experience higher levels of mortality in the winter than in the summer. A measure of this increase is provided on an annual basis, in the form of the excess winter mortality (EWM) figure and index (see Box One).

The elderly are more vulnerable than others during the winter and these figures are used to monitor health targets at a national and local level. Policies aimed at tackling EWM, such as winter fuel payments ${ }^{1}$ and influenza vaccinations, ${ }^{2}$ particularly focus on older people. Although EWM is associated with low temperatures, conditions directly relating to cold, such as hypothermia, are not the main cause of excess winter mortality. A previous article ${ }^{3}$ on EWM showed that circulatory and respiratory diseases exhibit marked seasonal fluctuations, with deaths from respiratory illnesses having the largest percentage seasonal increase.

\section{Results}

\section{Total excess winter mortality in England and Wales}

Figure 1 shows EWM in England and Wales, by year from the winter of $1950 / 51$ to $2008 / 09$. A five-year moving average is also presented to smooth out any short-term fluctuations. This shows that a sharp drop in EWM occurred between 1950 and 1970, with a more gradual decrease since then.

In the four months of winter 2008/09 there were an estimated 36,700 more deaths in England and Wales than in the non-winter period. There were just over 12,000 more excess winter deaths in 2008/09 than in 2007/08, an increase of 49 per cent. The last time EWM figures were higher than this was the winter of 1999/2000.

\section{Box one}

\section{Method for calculating excess winter mortality}

The current ONS standard method defines the winter period as December to March, and compares the number of deaths that occurred in this winter period with the average number of deaths occurring in the preceding August to November and the following April to July:

\section{EWM $=$ winter deaths - average non-winter deaths}

This produces a number of excess winter deaths that is rounded to the nearest 10 for final data and to the nearest 100 for provisional data. The EWM index is calculated so that comparisons can be made between sexes, age groups and regions, and is calculated as the number of excess winter deaths divided by the average non-winter deaths, expressed as a percentage:

EWM Index = (EWM / average non-winter deaths $) \times 100$

The EWM index is presented with 95 per cent confidence intervals in this report. 


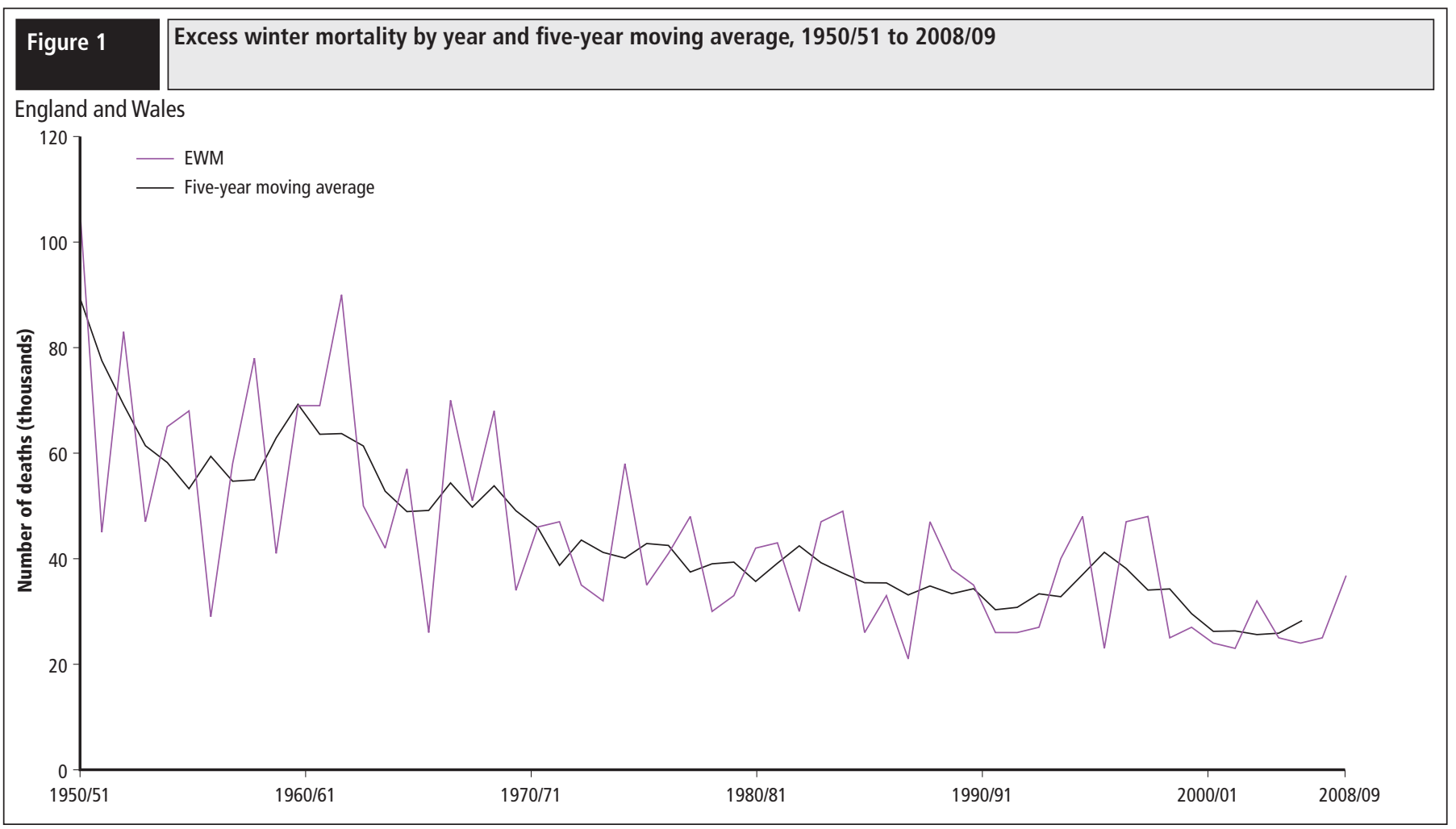

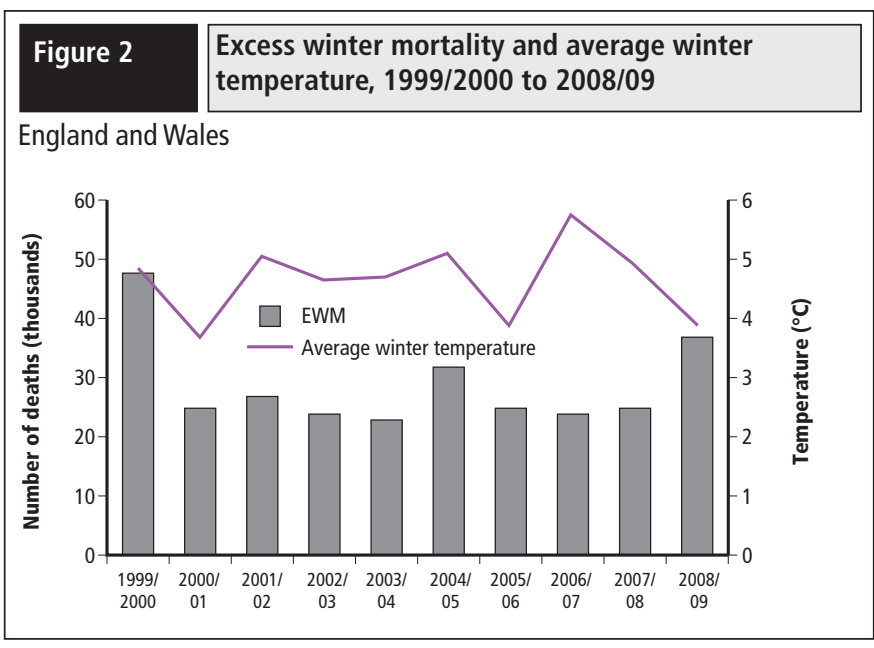

The number of extra deaths occurring in winter varies depending on temperature and the level of disease (particularly influenza) in the population, as well as other factors. ${ }^{3}$ Figure 2 shows that the increase in EWM between 2006/07 and 2008/09 corresponded with a decrease in the average temperature across the winter period. However, the relationship between temperature and EWM was less clear in other years.

When monthly mortality data for $2008 / 09$ were compared with the five-year average of deaths between 2003/04 and 2007/08 (Figure 3), deaths were higher in December 2008 to January 2009 than the fiveyear average. The mean monthly temperatures were generally lower throughout 2008/09 compared with the five-year average temperatures, with the largest difference occurring in January 2009. ${ }^{4}$

\section{Excess winter mortality by sex and age}

Figure 4 shows the number of excess winter deaths by sex and age in England and Wales for 2006/07 to 2008/09.
In 2008/09, there were 15,300 and 21,400 excess winter deaths in males and females respectively. The majority of these deaths occurred among those aged 75 and over in both sexes, although proportions varied by sex. Among those aged under 75, there were more excess winter deaths in males than females, with a male:female ratio of 1.29. In those aged 75 and over, there were more excess winter deaths in women than men, with a male:female ratio of 0.61 . A greater number, and higher proportion, of the female population are aged 75 and over ( 9 per cent compared with 6 per cent of males in 2008), which may wholly, or partially, account for the higher number of excess winter deaths in women.

In 2008/09, overall EWM for males was 44 per cent higher than in the winter of 2007/08. The largest increase was in males aged 75 to 84 , where the number increased by 59 per cent. For females in 2008/09, overall EWM increased by 52 per cent compared with 2007/08. This increase was reflected across all age groups.

\section{Excess winter mortality for Government Office Regions of England, and Wales}

Table 1 presents EWM and the EWM index by age and Government Office Region of England, and Wales. There are substantial year-on-year changes in the rank order of regions for EWM - for example, the North East had the lowest EWM index in the winter of 2008/09 and the highest EWM index for the previous winter of 2007/08. Research ${ }^{5}$ examining EWM among the elderly found little evidence for any consistent variation by geographical region within the UK.

Figure 5 shows the EWM index by Government Office Region of England, and Wales, for the winters of 2006/07 to 2008/09. The EWM index was significantly higher in 2008/09 than the previous two winters for all regions except the North East. The South East region had the highest EWM index for 2008/09 at 25.8, but Wales had the greatest percentage point increase between 2007/08 and 2008/09 (11.4 percentage points). The region with the lowest EWM index in 2008/09 was the North East - it was also the region with the smallest percentage point increase between 2007/08 and 2008/09 (1.9 percentage points). 


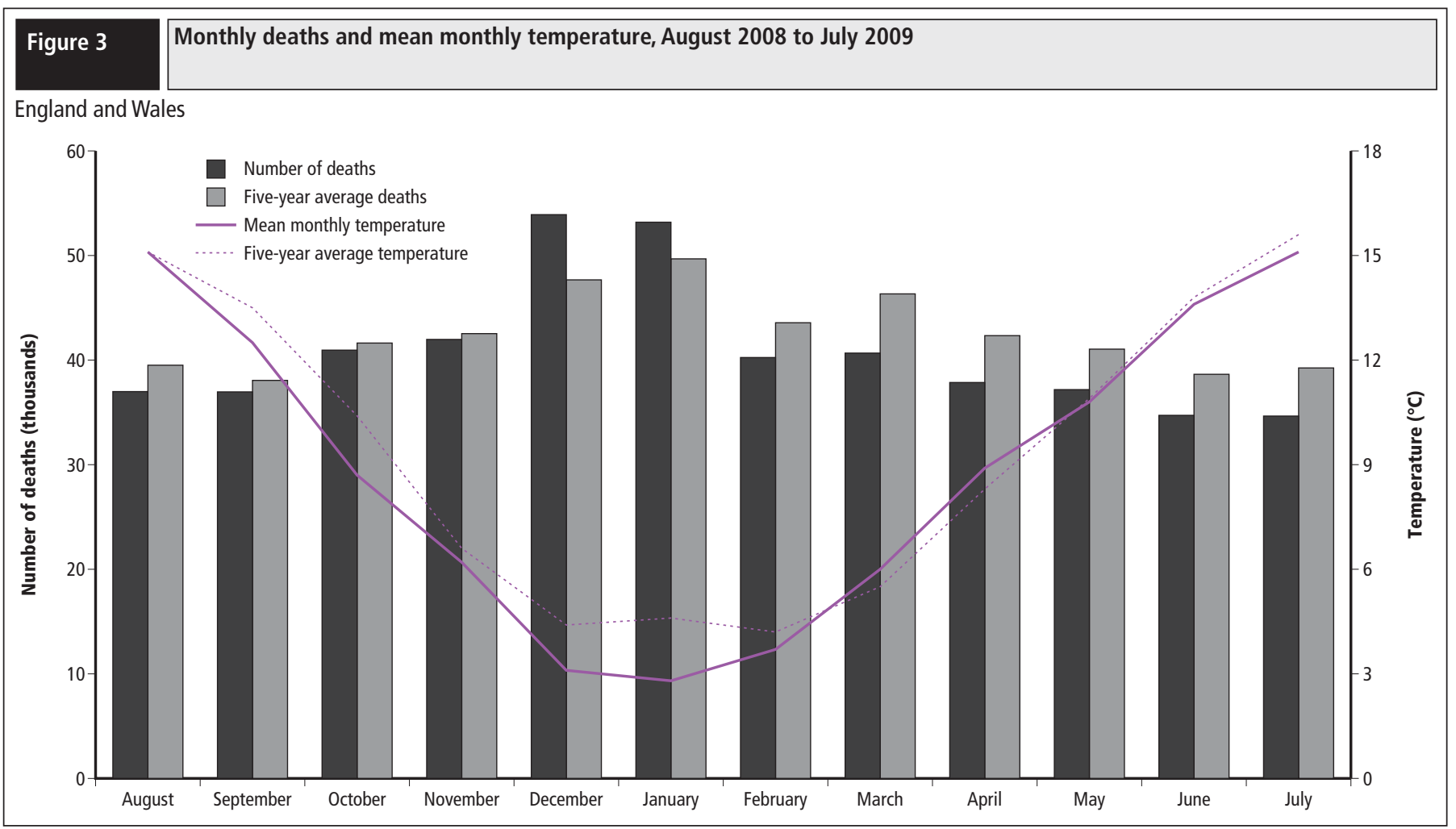

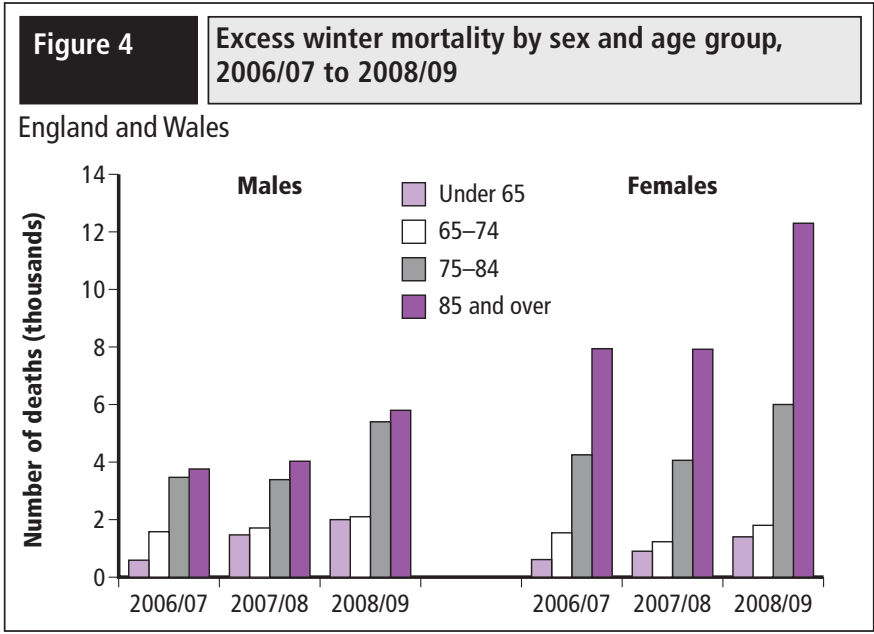

\section{Excess winter mortality by underlying cause of death}

Figure 6 shows EWM and the EWM index for circulatory diseases, respiratory diseases, external causes of injury and poisoning, and cancers (all neoplasms) for the winters of 2005/06 to 2007/08. Although cancers account for a quarter of all deaths annually, previous research ${ }^{3}$ found that there was no clear seasonal pattern for these deaths, which accounts for the low EWM and EWM index for cancers seen in Figure 6. Injury and poisoning deaths, however, include accidental falls, which can be affected by wintry conditions - for example, icy pavements - and the EWM index was 8.6 per cent in 2007/08, compared with 3.9 and 4.7 per cent in 2006/07 and 2005/06 respectively.

There were slightly more excess winter deaths with an underlying cause of circulatory disease than respiratory disease for the winters of 2005/06 and 2007/08, but a higher number of excess winter respiratory disease deaths in 2006/07 (Figure 6). However, the EWM index is much lower for circulatory diseases than for respiratory diseases for all of the winter periods. This means that a greater number of circulatory disease deaths than respiratory disease deaths occurred during the non-winter periods of $2005 / 06$ to $2007 / 08$.

For all of the causes examined except respiratory diseases, both the number of excess winter deaths and the EWM index increased between $2006 / 07$ and 2007/08. The number of excess winter deaths due to respiratory diseases decreased by 6 per cent in 2007/08, compared with 2006/07. Nevertheless, respiratory disease deaths still had the highest EWM index, compared with other causes for all of the winters analysed. In the winter of 2007/08, the EWM index for respiratory diseases was 42 per cent. Deaths from circulatory diseases and cancers showed slight fluctuations over the three winter periods. Injury and poisoning deaths showed the greatest change over this time period, with deaths more than doubling from 2006/07 to 2007/08. However, although deaths from injury and poisoning were significantly higher in 2007/08 compared with $2005 / 06$ and 2006/07, they are in line with figures from 2004/05.

Table 2 shows the number of excess winter deaths and the EWM index by sex and age group for circulatory diseases, respiratory diseases, injury and poisoning, and cancers in England and Wales for the winters of $2005 / 06$ to $2007 / 08$. For circulatory diseases, people aged 85 and over had the highest EWM index in both males and females across the winter periods and it was consistently higher when compared with the $0-64$ age group.

For respiratory diseases, people aged 85 and over also had the highest EWM index for males and females for the three winter periods examined. The only exception was for females in 2007/08 where the index was 52 per cent in the 0-64 age group and 49 percent in the 85 and over age group. The link between EWM and age, however, is not as strong as it is for circulatory diseases.

For deaths from injury and poisoning, the EWM index showed a clear sex difference: females have higher EWM than males. This sex difference is most marked in the younger age groups, as a greater 


\section{Table 1} Excess winter mortality by age group and usual residence of deceased, 2004/05 to 2008/09

Government Office Regions of England, Wales

\begin{tabular}{|c|c|c|c|c|c|c|c|c|c|c|c|}
\hline & & \multicolumn{2}{|c|}{$2004 / 05$} & \multicolumn{2}{|c|}{$2005 / 06$} & \multicolumn{2}{|c|}{$2006 / 07$} & \multicolumn{2}{|c|}{$2007 / 08$} & \multicolumn{2}{|c|}{$2008 / 09$} \\
\hline & & EWM $^{1}$ & EWM Index & EWM $^{1}$ & EWM Index & EWM $^{1}$ & EWM Index & EWM $^{1}$ & EWM Index & $\mathrm{EWM}^{2}$ & EWM Index \\
\hline \multirow[t]{5}{*}{ England and Wales ${ }^{3}$} & $0-64$ & 2,460 & 8.7 & 1,960 & 6.9 & 1,190 & 4.2 & 2,370 & 8.5 & 3,400 & 13.1 \\
\hline & $65-74$ & 4,000 & 14.1 & 2,930 & 10.7 & 3,120 & 11.9 & 2,930 & 11.3 & 3,900 & 15.4 \\
\hline & $75-84$ & 10,810 & 19.7 & 8,030 & 15.3 & 7,730 & 15.3 & 7,450 & 14.9 & 11,300 & 23.5 \\
\hline & $85+$ & 14,380 & 28.3 & 12,340 & 23.7 & 11,700 & 22.2 & 11,940 & 21.7 & 18,100 & 32.8 \\
\hline & All ages & 31,640 & 19.5 & 25,270 & 15.8 & 23,740 & 15.0 & 24,690 & 15.6 & 36,700 & 23.8 \\
\hline \multirow[t]{5}{*}{ England } & $0-64$ & 2,320 & 8.8 & 1,760 & 6.7 & 1,120 & 4.2 & 2,320 & 9.0 & 3,100 & 13.1 \\
\hline & $65-74$ & 3,700 & 14.1 & 2,750 & 10.8 & 2,940 & 12.1 & 2,810 & 11.7 & 3,700 & 15.4 \\
\hline & $75-84$ & 10,150 & 19.8 & 7,580 & 15.4 & 7,280 & 15.4 & 6,890 & 14.8 & 10,500 & 23.2 \\
\hline & $85+$ & 13,570 & 28.5 & 11,650 & 23.8 & 11,050 & 22.4 & 11,260 & 21.8 & 17,000 & 32.9 \\
\hline & All ages & 29,740 & 19.6 & 23,740 & 15.8 & 22,380 & 15.2 & 23,290 & 15.7 & 34,200 & 23.7 \\
\hline \multirow[t]{5}{*}{ Wales } & $0-64$ & 140 & 8.0 & 200 & 11.9 & 100 & 5.5 & 70 & 3.9 & 300 & 16.0 \\
\hline & $65-74$ & 300 & 16.2 & 210 & 11.6 & 210 & 12.4 & 130 & 7.6 & 300 & 16.5 \\
\hline & $75-84$ & 670 & 18.9 & 460 & 13.8 & 460 & 14.2 & 560 & 17.6 & 900 & 28.3 \\
\hline & $85+$ & 810 & 26.3 & 690 & 22.3 & 640 & 19.5 & 680 & 20.1 & 1,100 & 32.8 \\
\hline & All ages & 1,930 & 18.8 & 1,560 & 15.7 & 1,400 & 14.1 & 1,440 & 14.3 & 2,500 & 25.7 \\
\hline \multirow[t]{5}{*}{ North East } & $0-64$ & 50 & 3.3 & 120 & 8.1 & 90 & 5.4 & 210 & 14.1 & - & 2.8 \\
\hline & $65-74$ & 210 & 11.5 & 140 & 8.3 & 370 & 24.2 & 200 & 13.1 & 200 & 14.0 \\
\hline & $75-84$ & 480 & 15.2 & 350 & 11.8 & 540 & 19.4 & 430 & 14.9 & 700 & 24.0 \\
\hline & $85+$ & 730 & 31.7 & 470 & 19.3 & 510 & 20.7 & 670 & 26.6 & 700 & 28.5 \\
\hline & All ages & 1,460 & 16.6 & 1,080 & 12.6 & 1,510 & 18.0 & 1,500 & 17.9 & 1,600 & 19.8 \\
\hline \multirow[t]{5}{*}{ North West } & $0-64$ & 520 & 12.1 & 260 & 6.0 & 150 & 3.5 & 260 & 6.1 & 600 & 16.8 \\
\hline & $65-74$ & 750 & 17.7 & 300 & 7.3 & 490 & 12.1 & 410 & 10.3 & 500 & 13.5 \\
\hline & $75-84$ & 1,520 & 19.9 & 1,140 & 15.5 & 1,070 & 15.2 & 910 & 13.1 & 1,600 & 23.3 \\
\hline & $85+$ & 1,650 & 25.8 & 1,300 & 19.6 & 1,710 & 25.6 & 1,630 & 23.3 & 2,300 & 33.2 \\
\hline & All ages & 4,440 & 19.7 & 3,000 & 13.4 & 3,430 & 15.5 & 3,210 & 14.5 & 5,000 & 23.5 \\
\hline \multirow[t]{5}{*}{ Yorkshire and The Humber } & $0-64$ & 260 & 9.1 & 240 & 8.7 & 150 & 5.3 & 270 & 9.4 & 200 & 6.8 \\
\hline & $65-74$ & 410 & 14.2 & 240 & 8.7 & 340 & 12.8 & 270 & 9.9 & 400 & 15.2 \\
\hline & $75-84$ & 1,270 & 23.6 & 580 & 10.9 & 770 & 14.9 & 820 & 16.1 & 1,200 & 24.7 \\
\hline & $85+$ & 1,320 & 27.5 & 1,010 & 20.6 & 1,070 & 21.0 & 1,090 & 20.8 & 1,700 & 32.9 \\
\hline & All ages & 3,250 & 20.5 & 2,070 & 13.1 & 2,330 & 14.7 & 2,450 & 15.4 & 3,500 & 22.7 \\
\hline \multirow[t]{5}{*}{ East Midlands } & $0-64$ & 140 & 5.8 & 170 & 7.5 & 120 & 5.0 & 110 & 4.9 & 200 & 10.2 \\
\hline & $65-74$ & 340 & 14.4 & 240 & 10.6 & 270 & 12.4 & 180 & 8.3 & 400 & 17.3 \\
\hline & $75-84$ & 860 & 18.9 & 690 & 15.4 & 640 & 14.9 & 660 & 15.7 & 800 & 19.6 \\
\hline & $85+$ & 1,130 & 28.0 & 880 & 20.6 & 980 & 23.1 & 810 & 18.0 & 1,500 & 34.6 \\
\hline & All ages & 2,460 & 18.6 & 1,990 & 14.9 & 2,010 & 15.4 & 1,770 & 13.4 & 2,900 & 22.8 \\
\hline West Midlands & $0-64$ & 290 & 9.7 & 50 & 1.7 & 180 & 5.9 & 270 & 9.2 & 400 & 14.0 \\
\hline & $65-74$ & 430 & 14.2 & 490 & 17.0 & 400 & 14.3 & 460 & 16.9 & 400 & 16.3 \\
\hline & $75-84$ & 1,240 & 21.7 & 960 & 17.5 & 1,020 & 19.3 & 850 & 16.5 & 1,300 & 26.2 \\
\hline & $85+$ & 1,630 & 33.0 & 1,210 & 23.6 & 1,060 & 20.5 & 1,210 & 22.5 & 1,700 & 32.1 \\
\hline & All ages & 3,590 & 21.5 & 2,710 & 16.3 & 2,670 & 16.3 & 2,790 & 17.2 & 3,900 & 24.5 \\
\hline East of England & $0-64$ & 210 & 8.2 & 240 & 9.2 & 150 & 5.5 & 330 & 12.9 & 400 & 18.4 \\
\hline & $65-74$ & 420 & 15.6 & 350 & 13.5 & 260 & 10.4 & 240 & 9.5 & 300 & 11.8 \\
\hline & $75-84$ & 1,120 & 19.7 & 820 & 15.0 & 860 & 16.2 & 800 & 15.4 & 1,200 & 22.7 \\
\hline & $85+$ & 1,540 & 27.9 & 1,400 & 24.1 & 1,310 & 22.9 & 1,380 & 23.0 & 1,900 & 30.4 \\
\hline & All ages & 3,290 & 20.0 & 2,810 & 17.0 & 2,570 & 15.9 & 2,760 & 16.9 & 3,800 & 23.3 \\
\hline London & $0-64$ & 370 & 10.5 & 260 & 7.4 & 120 & 3.5 & 390 & 12.0 & 500 & 14.9 \\
\hline & $65-74$ & 340 & 11.5 & 230 & 8.2 & 220 & 8.1 & 290 & 11.1 & 500 & 20.1 \\
\hline & $75-84$ & 1,150 & 21.5 & 770 & 15.1 & 650 & 13.5 & 840 & 17.9 & 1,100 & 24.5 \\
\hline & $85+$ & 1,580 & 31.3 & 1,300 & 26.1 & 1,080 & 21.8 & 1,210 & 23.5 & 1,700 & 33.6 \\
\hline & All ages & 3,440 & 20.4 & 2,560 & 15.6 & 2,070 & 13.0 & 2,730 & 17.4 & 3,800 & 25.0 \\
\hline South East & $0-64$ & 310 & 8.2 & 260 & 6.8 & - & 0.1 & 250 & 6.6 & 600 & 15.8 \\
\hline & $65-74$ & 430 & 11.2 & 570 & 15.6 & 250 & 7.2 & 360 & 10.2 & 500 & 15.2 \\
\hline & $75-84$ & 1,500 & 18.4 & 1,470 & 19.2 & 1,080 & 14.8 & 930 & 12.7 & 1,700 & 24.7 \\
\hline & $85+$ & 2,350 & 27.4 & 2,380 & 27.3 & 1,690 & 19.2 & 1,980 & 21.5 & 3,200 & 34.4 \\
\hline & All ages & 4,590 & 18.8 & 4,680 & 19.6 & 3,030 & 12.9 & 3,520 & 14.8 & 6,000 & 25.8 \\
\hline South West & $0-64$ & 170 & 7.0 & 160 & 6.4 & 160 & 6.3 & 230 & 9.3 & 200 & 10.5 \\
\hline & $65-74$ & 380 & 15.0 & 180 & 7.1 & 330 & 13.8 & 390 & 17.1 & 400 & 16.3 \\
\hline & $75-84$ & 1,010 & 17.8 & 810 & 14.9 & 650 & 12.4 & 650 & 12.6 & 1,000 & 18.9 \\
\hline & $85+$ & 1,650 & 27.0 & 1,700 & 28.3 & 1,640 & 26.2 & 1,290 & 19.4 & 2,200 & 33.3 \\
\hline & All ages & 3,220 & 19.2 & 2,840 & 17.3 & 2,780 & 16.9 & 2,560 & 15.5 & 3,800 & 23.3 \\
\hline
\end{tabular}

1 Rounded to the nearest 10.

2 Provisional, rounded to the nearest 100

3 England and Wales data include non-residents who died in England or Wales. Data for England and Wales separately excludes deaths of non-residents. 

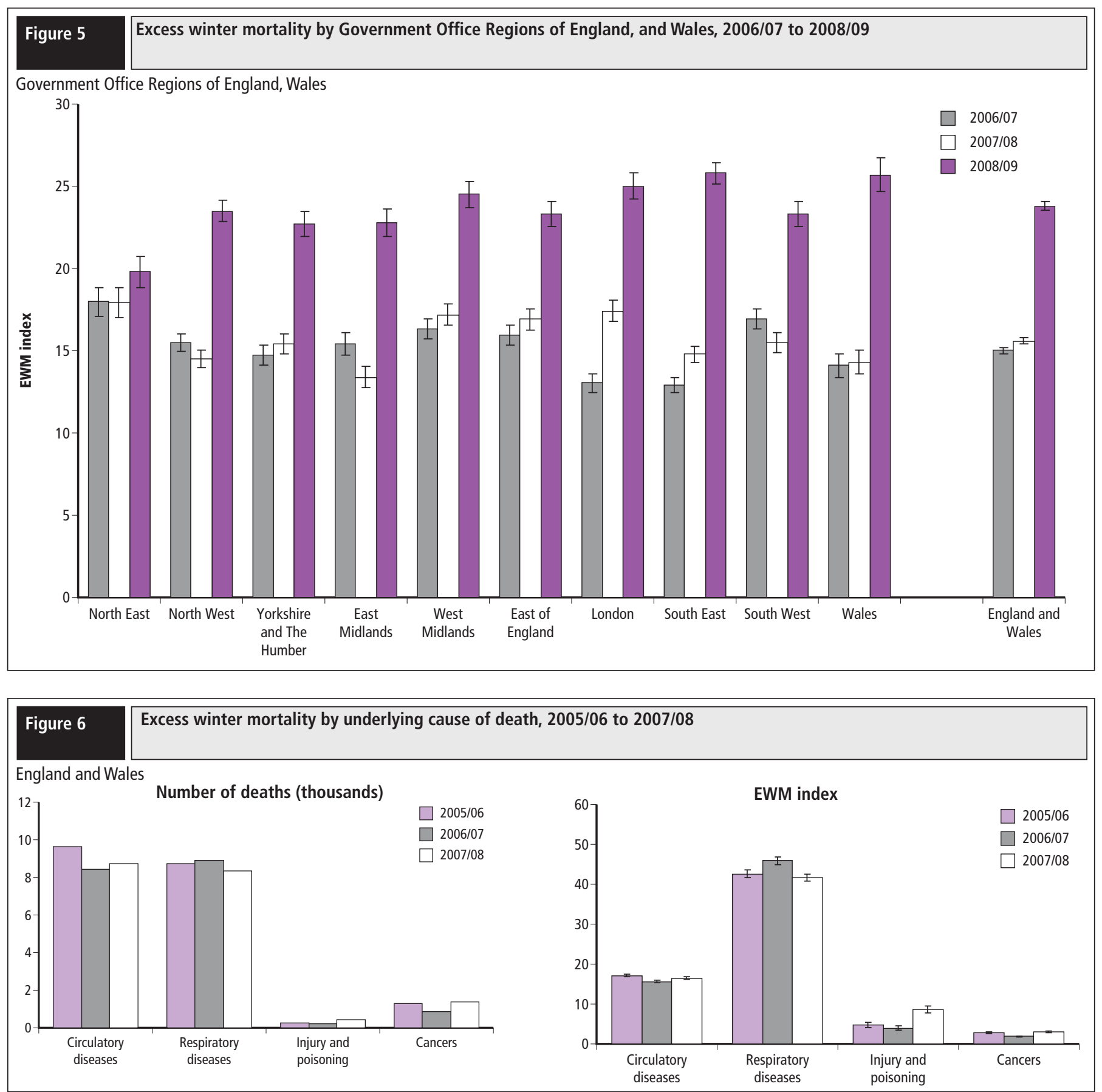

number of younger men aged 0 to 64 died from injury or poisoning in the summer months, compared with the winter months, but this pattern was reversed in females.

\section{Methods}

Excess winter mortality (EWM) is calculated by comparing the number of deaths occurring in winter with the number occurring in a non-winter period. Previous analysis compared methods of calculating EWM using different winter and non-winter periods. ${ }^{3}$ The method for calculating EWM used in this report can be found in Box One.

Mortality data are collected from death registration. Most deaths (almost 95 per cent) are registered within one month of the date of occurrence, although violent or unexpected deaths, which need further investigation from a coroner, can take much longer. So that timely EWM figures can be produced, ONS generates a special extract of mortality data in September for deaths that were registered by this month, but which occurred up to the end of July. These figures are then adjusted using the number of deaths from the previous year's extract, compared with the final number of deaths. This produces a provisional estimated number of deaths for January to July in the current year so that EWM can be calculated for the previous winter. As these figures are provisional they are rounded to the nearest 100 and are not produced for areas smaller than Government Office Region of England.

Cause of death figures have been produced using final figures (2005/062007/08). Appendix Tables A1 and A2 at the end of this report contain mortality data by age group, sex, area of usual residence (Government Office Region of England, and Wales) and month of occurrence from January to December 2007. Appendix Tables A3 and A4 present equivalent data for January to December 2008. 


\section{References}

1. Directgov - Information about winter fuel payments, accessed on 23 October 2009, available at:

www.direct.gov.uk/en/Pensionsandretirementplanning/Benefits/ BenefitsInRetirement/DG_10018657

2. Department of Health (2009) - Information about the seasonal flu vaccine, accessed on 23 October 2009, available at: www.immunisation.nhs.uk/Vaccines/Flu
3. Johnson H and Griffiths C (2003) 'Estimating excess winter mortality in England and Wales', Health Statistics Quarterly 20, 19-24. Available on the Office for National Statistics website at: www.statistics.gov.uk/statbase/Product.asp?vlnk $=6725$

4. The Met Office - UK climate summary, accessed on 23 October 2009, available at: www.metoffice.gov.uk/climate/uk/

5. Wilkinson P, Pattenden S, Armstrong B et al (2004) 'Vulnerability to winter mortality in elderly people in Britain: population based study'. British Medical Journal Sept 2004, 18, 329, 647-52. 
England and Wales

\begin{tabular}{|c|c|c|c|c|c|c|c|}
\hline & & \multicolumn{2}{|c|}{$2005 / 06$} & \multicolumn{2}{|c|}{$2006 / 07$} & \multicolumn{2}{|c|}{$2007 / 08$} \\
\hline & & $\mathrm{EWM}^{1}$ & EWM Index & $\mathrm{EWM}^{1}$ & EWM Index & $\mathrm{EWM}^{1}$ & EWM Index \\
\hline \multicolumn{8}{|l|}{ Males } \\
\hline Circulatory diseases & $0-64$ & 620 & 13.6 & 260 & 5.6 & 520 & 11.9 \\
\hline \multirow[t]{4}{*}{ (ICD-10 I00-I99) } & $65-74$ & 720 & 12.9 & 710 & 13.7 & 670 & 13.6 \\
\hline & $75-84$ & 1,750 & 17.4 & 1,220 & 12.7 & 1,400 & 15.1 \\
\hline & $85+$ & 1,500 & 22.3 & 1,490 & 21.8 & 1,450 & 20.6 \\
\hline & All ages & 4,600 & 17.0 & 3,670 & 14.0 & 4,040 & 15.8 \\
\hline Respiratory diseases & $0-64$ & 340 & 36.5 & 280 & 30.0 & 380 & 41.6 \\
\hline \multirow[t]{4}{*}{ (ICD-10 J00-J99) } & $65-74$ & 570 & 34.9 & 540 & 35.3 & 470 & 29.2 \\
\hline & $75-84$ & 1,180 & 31.6 & 1,250 & 36.4 & 1,180 & 34.1 \\
\hline & $85+$ & 1,360 & 43.0 & 1,370 & 42.8 & 1,420 & 42.1 \\
\hline & All ages & 3,460 & 36.4 & 3,440 & 37.8 & 3,460 & 36.9 \\
\hline Injury and poisoning & $0-64$ & -110 & -5.0 & -130 & -5.7 & -10 & -0.5 \\
\hline \multirow[t]{4}{*}{ (ICD-10 V01-Y89) } & $65-74$ & 10 & 3.4 & - & 0.4 & 30 & 9.8 \\
\hline & $75-84$ & 40 & 10.5 & 80 & 20.1 & 80 & 18.6 \\
\hline & $85+$ & 80 & 23.8 & 50 & 13.2 & 60 & 14.9 \\
\hline & All ages & 20 & 0.5 & - & 0.1 & 150 & 4.6 \\
\hline Cancers & $0-64$ & 160 & 2.9 & -130 & -2.4 & 170 & 3.1 \\
\hline \multirow[t]{4}{*}{ (ICD-10 C00-D48) } & $65-74$ & 70 & 1.0 & 60 & 1.0 & 290 & 4.5 \\
\hline & $75-84$ & 190 & 2.3 & 250 & 3.1 & 120 & 1.5 \\
\hline & $85+$ & 140 & 3.9 & 160 & 4.4 & 330 & 8.4 \\
\hline & All ages & 550 & 2.3 & 350 & 1.4 & 910 & 3.8 \\
\hline \multirow[t]{5}{*}{ All causes } & $0-64$ & 1,110 & 6.5 & 590 & 3.4 & 1,470 & 8.7 \\
\hline & $65-74$ & 1,540 & 9.6 & 1,580 & 10.3 & 1,710 & 11.2 \\
\hline & $75-84$ & 3,960 & 15.0 & 3,470 & 13.6 & 3,390 & 13.4 \\
\hline & $85+$ & 4,000 & 23.3 & 3,760 & 21.0 & 4,030 & 21.5 \\
\hline & All ages & 10,620 & 13.8 & 9,400 & 12.3 & 10,590 & 13.9 \\
\hline \multicolumn{8}{|l|}{ Females } \\
\hline Circulatory diseases & $0-64$ & 160 & 8.8 & 170 & 9.9 & 260 & 15.3 \\
\hline \multirow[t]{4}{*}{ (ICD-10 I00-I99) } & $65-74$ & 460 & 14.3 & 470 & 16.0 & 390 & 14.3 \\
\hline & $75-84$ & 1,540 & 15.5 & 1,420 & 15.6 & 1,500 & 17.1 \\
\hline & $85+$ & 2,890 & 20.2 & 2,700 & 19.4 & 2,560 & 17.9 \\
\hline & All ages & 5,040 & 17.3 & 4,750 & 17.2 & 4,700 & 17.1 \\
\hline Respiratory diseases & $0-64$ & 320 & 49.3 & 260 & 40.6 & 340 & 52.0 \\
\hline \multirow[t]{4}{*}{ (ICD-10 J00-J99) } & $65-74$ & 470 & 39.1 & 610 & 54.6 & 500 & 44.3 \\
\hline & $75-84$ & 1,480 & 40.9 & 1,580 & 47.3 & 1,310 & 39.5 \\
\hline & $85+$ & 2,990 & 54.4 & 3,020 & 57.9 & 2,750 & 49.2 \\
\hline & All ages & 5,260 & 48.0 & 5,470 & 53.1 & 4,910 & 45.8 \\
\hline Injury and poisoning & $0-64$ & 40 & 5.1 & 10 & 1.5 & 60 & 8.7 \\
\hline \multirow[t]{4}{*}{ (ICD-10 V01-Y89) } & $65-74$ & 20 & 10.7 & 10 & 4.5 & 20 & 14.1 \\
\hline & $75-84$ & 40 & 8.0 & 80 & 19.0 & 50 & 12.2 \\
\hline & $85+$ & 150 & 19.4 & 110 & 14.5 & 170 & 21.5 \\
\hline & All ages & 230 & 11.4 & 210 & 10.4 & 300 & 14.8 \\
\hline Cancers & $0-64$ & 40 & 0.7 & -100 & -1.9 & -20 & -0.4 \\
\hline \multirow[t]{4}{*}{ (ICD-10 C00-D48) } & $65-74$ & 290 & 6.0 & 160 & 3.3 & 80 & 1.6 \\
\hline & $75-84$ & 190 & 2.6 & 290 & 4.1 & 270 & 3.8 \\
\hline & $85+$ & 220 & 4.9 & 160 & 3.4 & 160 & 3.3 \\
\hline & All ages & 730 & 3.4 & 510 & 2.3 & 490 & 2.2 \\
\hline \multirow[t]{5}{*}{ All causes } & $0-64$ & 840 & 7.6 & 610 & 5.5 & 900 & 8.2 \\
\hline & $65-74$ & 1,390 & 12.4 & 1,540 & 14.2 & 1,230 & 11.4 \\
\hline & $75-84$ & 4,080 & 15.5 & 4,250 & 17.0 & 4,060 & 16.5 \\
\hline & $85+$ & 8,340 & 24.0 & 7,940 & 22.8 & 7,920 & 21.8 \\
\hline & All ages & 14,640 & 17.6 & 14,340 & 17.6 & 14,100 & 17.1 \\
\hline
\end{tabular}

1 Rounded to the nearest 10. 


\section{Appendix}

\begin{tabular}{|c|c|c|c|c|c|c|c|c|c|c|c|c|c|c|}
\hline \multirow{3}{*}{\multicolumn{2}{|c|}{$\begin{array}{l}\text { Table A1 Number of deat } \\
\text { Government Office Regions of England, Wale } \\
\end{array}$}} & \multicolumn{8}{|c|}{ hs by month, age group and area of usual residence, males, 2007} & \multicolumn{2}{|l|}{ 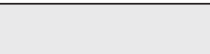 } & \\
\hline & & & & & & & & & & & & & & \\
\hline & & January & February & March & April & May & June & July & August & September & October & November & December & Total $^{1}$ \\
\hline \multirow[t]{5}{*}{ England, Wales and elsewhere } & $0-64$ & 4,735 & 4,393 & 4,511 & 4,480 & 4,516 & 4,230 & 4,299 & 4,110 & 4,080 & 4,324 & 4,179 & 4,617 & 52,479 \\
\hline & $65-74$ & 4,443 & 3,974 & 4,206 & 3,957 & 3,974 & 3,762 & 3,786 & 3,735 & 3,615 & 3,855 & 4,057 & 4,386 & 47,750 \\
\hline & $75-84$ & 7,444 & 7,241 & 7,175 & 6,620 & 6,537 & 6,118 & 6,241 & 6,402 & 5,963 & 6,533 & 6,654 & 7,499 & 80,428 \\
\hline & $85+$ & 5,586 & 5,508 & 5,367 & 4,765 & 4,641 & 4,373 & 4,465 & 4,387 & 4,240 & 4,724 & 5,022 & 5,884 & 58,962 \\
\hline & All ages & 22,208 & 21,116 & 21,259 & 19,822 & 19,668 & 18,483 & 18,791 & 18,634 & 17,898 & 19,436 & 19,912 & 22,386 & 239,619 \\
\hline North East & $0-64$ & 256 & 237 & 261 & 246 & 253 & 216 & 258 & 214 & 212 & 247 & 223 & 253 & 2,876 \\
\hline & $65-74$ & 280 & 231 & 271 & 220 & 217 & 225 & 215 & 204 & 203 & 192 & 231 & 255 & 2,744 \\
\hline & $75-84$ & 430 & 413 & 382 & 352 & 349 & 344 & 342 & 355 & 379 & 372 & 364 & 398 & 4,480 \\
\hline & $85+$ & 252 & 234 & 261 & 223 & 205 & 188 & 195 & 203 & 207 & 202 & 226 & 265 & 2,661 \\
\hline & All ages & 1,218 & 1,115 & 1,175 & 1,041 & 1,024 & 973 & 1,010 & 976 & 1,001 & 1,013 & 1,044 & 1,171 & 12,761 \\
\hline North West & $0-64$ & 711 & 683 & 657 & 689 & 711 & 618 & 623 & 621 & 625 & 620 & 634 & 713 & 7,905 \\
\hline & $65-74$ & 703 & 572 & 652 & 594 & 606 & 612 & 619 & 566 & 561 & 600 & 540 & 645 & 7,270 \\
\hline & $75-84$ & 1,062 & 989 & 963 & 902 & 884 & 838 & 876 & 873 & 830 & 887 & 949 & 958 & 11,011 \\
\hline & $85+$ & 717 & 720 & 658 & 563 & 551 & 540 & 523 & 504 & 512 & 569 & 624 & 742 & 7,223 \\
\hline & All ages & 3,193 & 2,964 & 2,930 & 2,748 & 2,752 & 2,608 & 2,641 & 2,564 & 2,528 & 2,676 & 2,747 & 3,058 & 33,409 \\
\hline Yorkshire and The Humber & $0-64$ & 511 & 442 & 452 & 463 & 449 & 426 & 416 & 433 & 422 & 429 & 438 & 508 & 5,389 \\
\hline & $65-74$ & 458 & 414 & 434 & 435 & 379 & 337 & 383 & 377 & 331 & 403 & 427 & 426 & 4,804 \\
\hline & $75-84$ & 714 & 712 & 700 & 655 & 662 & 633 & 607 & 665 & 563 & 644 & 625 & 770 & 7,950 \\
\hline & $85+$ & 515 & 508 & 492 & 461 & 415 & 412 & 391 & 403 & 379 & 427 & 452 & 516 & 5,371 \\
\hline & All ages & 2,198 & 2,076 & 2,078 & 2,014 & 1,905 & 1,808 & 1,797 & 1,878 & 1,695 & 1,903 & 1,942 & 2,220 & 23,514 \\
\hline East Midlands & $0-64$ & 372 & 376 & 360 & 371 & 364 & 326 & 315 & 327 & 345 & 361 & 363 & 360 & 4,240 \\
\hline & $65-74$ & 347 & 348 & 354 & 341 & 339 & 331 & 318 & 323 & 295 & 317 & 326 & 360 & 3,999 \\
\hline & $75-84$ & 601 & 635 & 641 & 559 & 596 & 507 & 500 & 536 & 500 & 515 & 599 & 639 & 6,828 \\
\hline & $85+$ & 469 & 438 & 468 & 389 & 394 & 370 & 371 & 374 & 354 & 394 & 435 & 511 & 4,967 \\
\hline & All ages & 1,789 & 1,797 & 1,823 & 1,660 & 1,693 & 1,534 & 1,504 & 1,560 & 1,494 & 1,587 & 1,723 & 1,870 & 20,034 \\
\hline West Midlands & $0-64$ & 519 & 474 & 504 & 490 & 472 & 481 & 424 & 433 & 437 & 481 & 450 & 509 & 5,674 \\
\hline & $65-74$ & 498 & 437 & 461 & 418 & 438 & 398 & 407 & 389 & 414 & 395 & 426 & 508 & 5,189 \\
\hline & $75-84$ & 799 & 807 & 803 & 673 & 699 & 673 & 673 & 648 & 599 & 670 & 703 & 805 & 8,552 \\
\hline & $85+$ & 535 & 523 & 515 & 434 & 478 & 451 & 420 & 443 & 428 & 480 & 456 & 578 & 5,741 \\
\hline & All ages & 2,351 & 2,241 & 2,283 & 2,015 & 2,087 & 2,003 & 1,924 & 1,913 & 1,878 & 2,026 & 2,035 & 2,400 & 25,156 \\
\hline East of England & $0-64$ & 451 & 388 & 425 & 407 & 417 & 391 & 442 & 392 & 390 & 387 & 387 & 430 & 4,910 \\
\hline & $65-74$ & 448 & 374 & 388 & 374 & 398 & 355 & 341 & 344 & 355 & 372 & 419 & 423 & 4,591 \\
\hline & $75-84$ & 814 & 777 & 750 & 716 & 659 & 624 & 653 & 677 & 632 & 723 & 658 & 809 & 8,492 \\
\hline & $85+$ & 629 & 601 & 569 & 571 & 519 & 457 & 503 & 485 & 480 & 493 & 552 & 604 & 6,463 \\
\hline & All ages & 2,342 & 2,140 & 2,132 & 2,068 & 1,993 & 1,827 & 1,939 & 1,898 & 1,857 & 1,975 & 2,016 & 2,266 & 24,456 \\
\hline London & $0-64$ & 568 & 548 & 553 & 506 & 542 & 561 & 532 & 501 & 483 & 462 & 489 & 556 & 6,301 \\
\hline & $65-74$ & 451 & 393 & 422 & 422 & 405 & 383 & 393 & 386 & 414 & 360 & 448 & 455 & 4,932 \\
\hline & $75-84$ & 710 & 702 & 641 & 643 & 604 & 584 & 640 & 597 & 590 & 601 & 666 & 695 & 7,673 \\
\hline & $85+$ & 541 & 477 & 529 & 472 & 462 & 426 & 440 & 399 & 382 & 451 & 504 & 580 & 5,663 \\
\hline & All ages & 2,270 & 2,120 & 2,145 & 2,043 & 2,013 & 1,954 & 2,005 & 1,883 & 1,869 & 1,874 & 2,107 & 2,286 & 24,569 \\
\hline South East & $0-64$ & 632 & 544 & 588 & 580 & 641 & 530 & 592 & 546 & 519 & 647 & 531 & 566 & 6,916 \\
\hline & $65-74$ & 580 & 509 & 550 & 515 & 532 & 509 & 512 & 506 & 506 & 542 & 575 & 645 & 6,481 \\
\hline & $75-84$ & 1,063 & 998 & 1,056 & 964 & 943 & 853 & 899 & 941 & 885 & 948 & 1,012 & 1,098 & 11,661 \\
\hline & $85+$ & 936 & 942 & 887 & 754 & 761 & 719 & 756 & 723 & 708 & 822 & 881 & 1,033 & 9,922 \\
\hline & All ages & 3,211 & 2,993 & 3,081 & 2,813 & 2,877 & 2,611 & 2,759 & 2,716 & 2,618 & 2,959 & 2,999 & 3,342 & 34,980 \\
\hline South West & $0-64$ & 411 & 386 & 408 & 400 & 374 & 383 & 393 & 351 & 356 & 373 & 350 & 389 & 4,574 \\
\hline & $65-74$ & 401 & 417 & 391 & 379 & 363 & 347 & 346 & 345 & 300 & 360 & 356 & 385 & 4,390 \\
\hline & $75-84$ & 775 & 723 & 785 & 712 & 667 & 655 & 662 & 655 & 613 & 750 & 650 & 781 & 8,428 \\
\hline & $85+$ & 661 & 734 & 671 & 574 & 564 & 551 & 570 & 568 & 527 & 583 & 618 & 722 & 7,343 \\
\hline & All ages & 2,248 & 2,260 & 2,255 & 2,065 & 1,968 & 1,936 & 1,971 & 1,919 & 1,796 & 2,066 & 1,974 & 2,277 & 24,735 \\
\hline Wales & $0-64$ & 278 & 281 & 261 & 298 & 269 & 261 & 271 & 256 & 261 & 274 & 287 & 304 & 3,303 \\
\hline & $65-74$ & 262 & 268 & 273 & 247 & 281 & 247 & 232 & 274 & 227 & 299 & 297 & 275 & 3,182 \\
\hline & $75-84$ & 468 & 480 & 446 & 432 & 467 & 399 & 375 & 441 & 366 & 406 & 424 & 533 & 5,237 \\
\hline & $85+$ & 325 & 327 & 314 & 319 & 290 & 254 & 291 & 281 & 259 & 298 & 272 & 330 & 3,560 \\
\hline & All ages & 1,333 & 1,356 & 1,294 & 1,296 & 1,307 & 1,161 & 1,169 & 1,252 & 1,113 & 1,277 & 1,280 & 1,442 & 15,282 \\
\hline England & $0-64$ & 4,431 & 4,078 & 4,208 & 4,152 & 4,223 & 3,932 & 3,995 & 3,818 & 3,789 & 4,007 & 3,865 & 4,284 & 48,785 \\
\hline & $65-74$ & 4,166 & 3,695 & 3,923 & 3,698 & 3,677 & 3,497 & 3,534 & 3,440 & 3,379 & 3,541 & 3,748 & 4,102 & 44,400 \\
\hline & $75-84$ & 6,968 & 6,756 & 6,721 & 6,176 & 6,063 & 5,711 & 5,852 & 5,947 & 5,591 & 6,110 & 6,226 & 6,953 & 75,075 \\
\hline & $85+$ & 5,255 & 5,177 & 5,050 & 4,441 & 4,349 & 4,114 & 4,169 & 4,102 & 3,977 & 4,421 & 4,748 & 5,551 & 55,354 \\
\hline & All ages & 20,820 & 19,706 & 19,902 & 18,467 & 18,312 & 17,254 & 17,550 & 17,307 & 16,736 & 18,079 & 18,587 & 20,890 & 223,614 \\
\hline England and Wales & $0-64$ & 4,709 & 4,359 & 4,469 & 4,450 & 4,492 & 4,193 & 4,266 & 4,074 & 4,050 & 4,281 & 4,152 & 4,588 & 52,088 \\
\hline & $65-74$ & 4,428 & 3,963 & 4,196 & 3,945 & 3,958 & 3,744 & 3,766 & 3,714 & 3,606 & 3,840 & 4,045 & 4,377 & 47,582 \\
\hline & $75-84$ & 7,436 & 7,236 & 7,167 & 6,608 & 6,530 & 6,110 & 6,227 & 6,388 & 5,957 & 6,516 & 6,650 & 7,486 & 80,312 \\
\hline & $85+$ & 5,580 & 5,504 & 5,364 & 4,760 & 4,639 & 4,368 & 4,460 & 4,383 & 4,236 & 4,719 & 5,020 & 5,881 & 58,914 \\
\hline & All ages & 22,153 & 21,062 & 21,196 & 19,763 & 19,619 & 18,415 & 18,719 & 18,559 & 17,849 & 19,356 & 19,867 & 22,332 & 238,896 \\
\hline elsewhere & $0-64$ & 26 & 34 & 42 & 30 & 24 & 37 & 33 & 36 & 30 & 43 & 27 & 29 & 391 \\
\hline & $65-74$ & 15 & 11 & 10 & 12 & 16 & 18 & 20 & 21 & 9 & 15 & 12 & 9 & 168 \\
\hline & $75-84$ & 8 & 5 & 8 & 12 & 7 & 8 & 14 & 14 & 6 & 17 & 4 & 13 & 116 \\
\hline & $85+$ & 6 & 4 & 3 & 5 & 2 & 5 & 5 & 4 & 4 & 5 & 2 & 3 & 48 \\
\hline & All ages & 55 & 54 & 63 & 59 & 49 & 68 & 72 & 75 & 49 & 80 & 45 & 54 & 723 \\
\hline
\end{tabular}


Government Office Regions of England, Wales

\begin{tabular}{|c|c|c|c|c|c|c|c|c|c|c|c|c|c|c|}
\hline & & January & February & March & April & May & June & July & August & September & October & November & December & Total $^{1}$ \\
\hline England, Wales and elsewhere & $\begin{array}{l}0-64 \\
65-74 \\
75-84 \\
85+ \\
\text { All ages }\end{array}$ & $\begin{array}{r}3,066 \\
3,251 \\
7,833 \\
11,213 \\
25,363\end{array}$ & $\begin{array}{r}2,795 \\
3,005 \\
7,196 \\
10,918 \\
23,914\end{array}$ & $\begin{array}{r}2,901 \\
3,054 \\
7,163 \\
10,472 \\
23,590\end{array}$ & $\begin{array}{r}2,853 \\
2,804 \\
6,609 \\
9,440 \\
21,706\end{array}$ & $\begin{array}{r}2,873 \\
2,751 \\
6,201 \\
9,059 \\
20,884\end{array}$ & $\begin{array}{r}2,696 \\
2,599 \\
5,973 \\
8,576 \\
19,844\end{array}$ & $\begin{array}{r}2,692 \\
2,523 \\
6,116 \\
8,607 \\
19,938\end{array}$ & $\begin{array}{r}2,683 \\
2,543 \\
5,965 \\
8,563 \\
19,754\end{array}$ & $\begin{array}{r}2,627 \\
2,579 \\
5,858 \\
8,341 \\
19,405\end{array}$ & $\begin{array}{r}2,770 \\
2,770 \\
6,373 \\
9,331 \\
21,244\end{array}$ & $\begin{array}{r}2,835 \\
2,823 \\
6,609 \\
9,733 \\
22,000\end{array}$ & $\begin{array}{r}3,038 \\
3,099 \\
7,490 \\
11,594 \\
25,221\end{array}$ & $\begin{array}{r}33,830 \\
33,801 \\
79,387 \\
115,847 \\
262,865\end{array}$ \\
\hline North East & $\begin{array}{l}0-64 \\
65-74 \\
75-84 \\
85+ \\
\text { All ages }\end{array}$ & $\begin{array}{r}173 \\
221 \\
434 \\
541 \\
1,369\end{array}$ & $\begin{array}{r}167 \\
185 \\
394 \\
468 \\
1,214\end{array}$ & $\begin{array}{r}166 \\
213 \\
433 \\
493 \\
1,305\end{array}$ & $\begin{array}{r}163 \\
161 \\
369 \\
444 \\
1,137\end{array}$ & $\begin{array}{r}158 \\
174 \\
363 \\
399 \\
1,094\end{array}$ & $\begin{array}{r}135 \\
172 \\
340 \\
379 \\
1,026\end{array}$ & $\begin{array}{r}140 \\
154 \\
341 \\
440 \\
1,075\end{array}$ & $\begin{array}{r}138 \\
152 \\
359 \\
397 \\
1,046\end{array}$ & $\begin{array}{r}125 \\
158 \\
352 \\
370 \\
1,005\end{array}$ & $\begin{array}{r}163 \\
152 \\
379 \\
444 \\
1,138\end{array}$ & $\begin{array}{r}145 \\
160 \\
388 \\
472 \\
1,165\end{array}$ & $\begin{array}{r}165 \\
197 \\
434 \\
533 \\
1,329\end{array}$ & $\begin{array}{r}1,838 \\
2,099 \\
4,586 \\
5,380 \\
13,903\end{array}$ \\
\hline North West & $\begin{array}{l}0-64 \\
65-74 \\
75-84 \\
85+ \\
\text { All ages }\end{array}$ & $\begin{array}{r}471 \\
507 \\
1,111 \\
1,475 \\
3,564\end{array}$ & $\begin{array}{r}383 \\
508 \\
1,030 \\
1,468 \\
3,389\end{array}$ & $\begin{array}{r}440 \\
504 \\
1,046 \\
1,408 \\
3,398\end{array}$ & $\begin{array}{r}412 \\
445 \\
970 \\
1,263 \\
3,090\end{array}$ & $\begin{array}{r}414 \\
430 \\
899 \\
1,155 \\
2,898\end{array}$ & $\begin{array}{r}371 \\
447 \\
855 \\
1,130 \\
2,803\end{array}$ & $\begin{array}{r}441 \\
383 \\
916 \\
1,084 \\
2,824\end{array}$ & $\begin{array}{r}400 \\
363 \\
853 \\
1,073 \\
2,689\end{array}$ & $\begin{array}{r}416 \\
363 \\
856 \\
1,138 \\
2,773\end{array}$ & $\begin{array}{r}413 \\
451 \\
923 \\
1,239 \\
3,026\end{array}$ & $\begin{array}{r}423 \\
473 \\
935 \\
1,277 \\
3,108\end{array}$ & $\begin{array}{r}453 \\
439 \\
1,028 \\
1,511 \\
3,431\end{array}$ & $\begin{array}{r}5,037 \\
5,313 \\
11,422 \\
15,221 \\
36,993\end{array}$ \\
\hline Yorkshire and The Humber & $\begin{array}{l}0-64 \\
65-74 \\
75-84 \\
85+ \\
\text { All ages }\end{array}$ & $\begin{array}{r}299 \\
361 \\
827 \\
1,097 \\
2,584\end{array}$ & $\begin{array}{r}321 \\
297 \\
785 \\
1,063 \\
2,466\end{array}$ & $\begin{array}{r}281 \\
297 \\
737 \\
1,012 \\
2,327\end{array}$ & $\begin{array}{r}314 \\
290 \\
708 \\
941 \\
2,253\end{array}$ & $\begin{array}{r}287 \\
296 \\
668 \\
868 \\
2,119\end{array}$ & $\begin{array}{r}269 \\
274 \\
624 \\
817 \\
1,984\end{array}$ & $\begin{array}{r}260 \\
287 \\
584 \\
874 \\
2,005\end{array}$ & $\begin{array}{r}279 \\
268 \\
614 \\
856 \\
2,017\end{array}$ & $\begin{array}{r}269 \\
301 \\
620 \\
836 \\
2,026\end{array}$ & $\begin{array}{r}271 \\
288 \\
662 \\
914 \\
2,135\end{array}$ & $\begin{array}{r}313 \\
288 \\
674 \\
935 \\
2,210\end{array}$ & $\begin{array}{r}350 \\
329 \\
820 \\
1,183 \\
2,682\end{array}$ & $\begin{array}{r}3,514 \\
3,576 \\
8,323 \\
11,396 \\
26,809\end{array}$ \\
\hline East Midlands & $\begin{array}{l}0-64 \\
65-74 \\
75-84 \\
85+ \\
\text { All ages }\end{array}$ & $\begin{array}{r}280 \\
272 \\
635 \\
880 \\
2,067\end{array}$ & $\begin{array}{r}242 \\
242 \\
632 \\
904 \\
2,020\end{array}$ & $\begin{array}{r}249 \\
243 \\
557 \\
837 \\
1,886\end{array}$ & $\begin{array}{r}231 \\
238 \\
534 \\
664 \\
1,667\end{array}$ & $\begin{array}{r}243 \\
234 \\
483 \\
744 \\
1,704\end{array}$ & $\begin{array}{r}215 \\
181 \\
496 \\
688 \\
1,580\end{array}$ & $\begin{array}{r}252 \\
196 \\
488 \\
689 \\
1,625\end{array}$ & $\begin{array}{r}214 \\
232 \\
486 \\
691 \\
1,623\end{array}$ & $\begin{array}{r}213 \\
209 \\
505 \\
676 \\
1,603\end{array}$ & $\begin{array}{r}236 \\
220 \\
553 \\
758 \\
1,767\end{array}$ & $\begin{array}{r}243 \\
228 \\
511 \\
761 \\
1,743\end{array}$ & $\begin{array}{r}258 \\
231 \\
641 \\
907 \\
2,037\end{array}$ & $\begin{array}{r}2,876 \\
2,726 \\
6,521 \\
9,199 \\
21,322\end{array}$ \\
\hline West Midlands & $\begin{array}{l}0-64 \\
65-74 \\
75-84 \\
85+ \\
\text { All ages }\end{array}$ & $\begin{array}{r}297 \\
346 \\
843 \\
1,054 \\
2,540\end{array}$ & $\begin{array}{r}306 \\
317 \\
796 \\
1,108 \\
2,527\end{array}$ & $\begin{array}{r}311 \\
334 \\
744 \\
1,071 \\
2,460\end{array}$ & $\begin{array}{r}294 \\
290 \\
701 \\
967 \\
2,252\end{array}$ & $\begin{array}{r}305 \\
282 \\
637 \\
880 \\
2,104\end{array}$ & $\begin{array}{r}288 \\
255 \\
671 \\
845 \\
2,059\end{array}$ & $\begin{array}{r}244 \\
271 \\
670 \\
804 \\
1,989\end{array}$ & $\begin{array}{r}267 \\
236 \\
612 \\
888 \\
2,003\end{array}$ & $\begin{array}{r}295 \\
270 \\
583 \\
770 \\
1,918\end{array}$ & $\begin{array}{r}285 \\
292 \\
650 \\
885 \\
2,112\end{array}$ & $\begin{array}{r}292 \\
286 \\
691 \\
934 \\
2,203\end{array}$ & $\begin{array}{r}307 \\
359 \\
766 \\
1,193 \\
2,625\end{array}$ & $\begin{array}{r}3,491 \\
3,538 \\
8,364 \\
11,399 \\
26,792\end{array}$ \\
\hline East of England & $\begin{array}{l}0-64 \\
65-74 \\
75-84 \\
85+ \\
\text { All ages }\end{array}$ & $\begin{array}{r}299 \\
282 \\
831 \\
1,204 \\
2,616\end{array}$ & $\begin{array}{r}260 \\
280 \\
705 \\
1,185 \\
2,430\end{array}$ & $\begin{array}{r}290 \\
280 \\
731 \\
1,115 \\
2,416\end{array}$ & $\begin{array}{r}270 \\
266 \\
632 \\
1,007 \\
2,175\end{array}$ & $\begin{array}{r}272 \\
286 \\
655 \\
978 \\
2,191\end{array}$ & $\begin{array}{r}272 \\
248 \\
583 \\
936 \\
2,039\end{array}$ & $\begin{array}{r}242 \\
247 \\
627 \\
929 \\
2,045\end{array}$ & $\begin{array}{r}229 \\
280 \\
644 \\
887 \\
2,040\end{array}$ & $\begin{array}{r}230 \\
239 \\
625 \\
889 \\
1,983\end{array}$ & $\begin{array}{r}248 \\
270 \\
619 \\
1,051 \\
2,188\end{array}$ & $\begin{array}{r}271 \\
270 \\
679 \\
1,058 \\
2,278\end{array}$ & $\begin{array}{r}279 \\
304 \\
813 \\
1,270 \\
2,666\end{array}$ & $\begin{array}{r}3,162 \\
3,252 \\
8,145 \\
12,509 \\
27,068\end{array}$ \\
\hline London & $\begin{array}{l}0-64 \\
65-74 \\
75-84 \\
85+ \\
\text { All ages }\end{array}$ & $\begin{array}{r}346 \\
326 \\
776 \\
1,054 \\
2,502\end{array}$ & $\begin{array}{r}300 \\
283 \\
649 \\
969 \\
2,201\end{array}$ & $\begin{array}{r}350 \\
306 \\
668 \\
966 \\
2,290\end{array}$ & $\begin{array}{r}326 \\
298 \\
607 \\
854 \\
2,085\end{array}$ & $\begin{array}{r}326 \\
268 \\
556 \\
907 \\
2,057\end{array}$ & $\begin{array}{r}335 \\
251 \\
569 \\
783 \\
1,938\end{array}$ & $\begin{array}{r}301 \\
238 \\
575 \\
799 \\
1,913\end{array}$ & $\begin{array}{r}331 \\
242 \\
544 \\
789 \\
1,906\end{array}$ & $\begin{array}{r}320 \\
275 \\
547 \\
738 \\
1,880\end{array}$ & $\begin{array}{r}309 \\
276 \\
594 \\
836 \\
2,015\end{array}$ & $\begin{array}{r}307 \\
301 \\
618 \\
917 \\
2,143\end{array}$ & $\begin{array}{r}339 \\
320 \\
706 \\
1,086 \\
2,451\end{array}$ & $\begin{array}{r}3,890 \\
3,384 \\
7,409 \\
10,698 \\
25,381\end{array}$ \\
\hline South East & $\begin{array}{l}0-64 \\
65-74 \\
75-84 \\
85+ \\
\text { All ages }\end{array}$ & $\begin{array}{r}396 \\
431 \\
1,098 \\
1,781 \\
3,706\end{array}$ & $\begin{array}{r}378 \\
384 \\
1,028 \\
1,780 \\
3,570\end{array}$ & $\begin{array}{r}374 \\
372 \\
1,006 \\
1,697 \\
3,449\end{array}$ & $\begin{array}{r}393 \\
364 \\
966 \\
1,581 \\
3,304\end{array}$ & $\begin{array}{r}422 \\
356 \\
932 \\
1,521 \\
3,231\end{array}$ & $\begin{array}{r}387 \\
360 \\
828 \\
1,482 \\
3,057\end{array}$ & $\begin{array}{r}387 \\
334 \\
917 \\
1,444 \\
3,082\end{array}$ & $\begin{array}{r}385 \\
372 \\
862 \\
1,452 \\
3,071\end{array}$ & $\begin{array}{r}366 \\
326 \\
814 \\
1,410 \\
2,916\end{array}$ & $\begin{array}{r}424 \\
413 \\
890 \\
1,521 \\
3,248\end{array}$ & $\begin{array}{r}376 \\
390 \\
994 \\
1,631 \\
3,391\end{array}$ & $\begin{array}{r}439 \\
424 \\
1,077 \\
1,952 \\
3,892\end{array}$ & $\begin{array}{r}4,727 \\
4,526 \\
11,412 \\
19,252 \\
39,917\end{array}$ \\
\hline South West & $\begin{array}{l}0-64 \\
65-74 \\
75-84 \\
85+ \\
\text { All ages }\end{array}$ & $\begin{array}{r}285 \\
281 \\
765 \\
1,388 \\
2,719\end{array}$ & $\begin{array}{r}245 \\
273 \\
702 \\
1,303 \\
2,523\end{array}$ & $\begin{array}{r}258 \\
296 \\
773 \\
1,262 \\
2,589\end{array}$ & $\begin{array}{r}255 \\
236 \\
683 \\
1,121 \\
2,295\end{array}$ & $\begin{array}{r}246 \\
210 \\
626 \\
1,038 \\
2,120\end{array}$ & $\begin{array}{r}241 \\
220 \\
601 \\
1,002 \\
2,064\end{array}$ & $\begin{array}{r}218 \\
227 \\
614 \\
993 \\
2,052\end{array}$ & $\begin{array}{r}247 \\
228 \\
632 \\
1,009 \\
2,116\end{array}$ & $\begin{array}{r}229 \\
236 \\
575 \\
1,000 \\
2,040\end{array}$ & $\begin{array}{r}250 \\
222 \\
697 \\
1,117 \\
2,286\end{array}$ & $\begin{array}{r}274 \\
237 \\
656 \\
1,154 \\
2,321\end{array}$ & $\begin{array}{r}265 \\
276 \\
735 \\
1,273 \\
2,549\end{array}$ & $\begin{array}{r}3,013 \\
2,942 \\
8,059 \\
13,660 \\
27,674\end{array}$ \\
\hline Wales & $\begin{array}{l}0-64 \\
65-74 \\
75-84 \\
85+ \\
\text { All ages }\end{array}$ & $\begin{array}{r}208 \\
218 \\
502 \\
730 \\
1,658\end{array}$ & $\begin{array}{r}182 \\
223 \\
469 \\
664 \\
1,538\end{array}$ & $\begin{array}{r}173 \\
204 \\
464 \\
609 \\
1,450\end{array}$ & $\begin{array}{r}182 \\
202 \\
425 \\
592 \\
1,401\end{array}$ & $\begin{array}{r}185 \\
204 \\
373 \\
567 \\
1,329\end{array}$ & $\begin{array}{r}164 \\
185 \\
399 \\
511 \\
1,259\end{array}$ & $\begin{array}{r}188 \\
172 \\
374 \\
549 \\
1,283\end{array}$ & $\begin{array}{r}172 \\
157 \\
355 \\
517 \\
1,201\end{array}$ & $\begin{array}{r}146 \\
190 \\
372 \\
509 \\
1,217\end{array}$ & $\begin{array}{r}160 \\
180 \\
395 \\
565 \\
1,300\end{array}$ & $\begin{array}{r}180 \\
184 \\
457 \\
591 \\
1,412\end{array}$ & $\begin{array}{r}168 \\
209 \\
463 \\
678 \\
1,518\end{array}$ & $\begin{array}{r}2,108 \\
2,328 \\
5,048 \\
7,082 \\
16,566\end{array}$ \\
\hline England & $\begin{array}{l}0-64 \\
65-74 \\
75-84 \\
85+ \\
\text { All ages }\end{array}$ & $\begin{array}{r}2,846 \\
3,027 \\
7,320 \\
10,474 \\
23,667\end{array}$ & $\begin{array}{r}2,602 \\
2,769 \\
6,721 \\
10,248 \\
22,340\end{array}$ & $\begin{array}{r}2,719 \\
2,845 \\
6,695 \\
9,861 \\
22,120\end{array}$ & $\begin{array}{r}2,658 \\
2,588 \\
6,170 \\
8,842 \\
20,258\end{array}$ & $\begin{array}{r}2,673 \\
2,536 \\
5,819 \\
8,490 \\
19,518\end{array}$ & $\begin{array}{r}2,513 \\
2,408 \\
5,567 \\
8,062 \\
18,550\end{array}$ & $\begin{array}{r}2,485 \\
2,337 \\
5,732 \\
8,056 \\
18,610\end{array}$ & $\begin{array}{r}2,490 \\
2,373 \\
5,606 \\
8,042 \\
18,511\end{array}$ & $\begin{array}{r}2,463 \\
2,377 \\
5,477 \\
7,827 \\
18,144\end{array}$ & $\begin{array}{r}2,599 \\
2,584 \\
5,967 \\
8,765 \\
19,915\end{array}$ & $\begin{array}{r}2,644 \\
2,633 \\
6,146 \\
9,139 \\
20,562\end{array}$ & $\begin{array}{r}2,855 \\
2,879 \\
7,020 \\
10,908 \\
23,662\end{array}$ & $\begin{array}{r}31,548 \\
31,356 \\
74,241 \\
108,714 \\
245,859\end{array}$ \\
\hline England and Wales & $\begin{array}{l}0-64 \\
65-74 \\
75-84 \\
85+ \\
\text { All ages }\end{array}$ & $\begin{array}{r}3,054 \\
3,245 \\
7,822 \\
11,204 \\
25,325\end{array}$ & $\begin{array}{r}2,784 \\
2,992 \\
7,190 \\
10,912 \\
23,878\end{array}$ & $\begin{array}{r}2,892 \\
3,049 \\
7,159 \\
10,470 \\
23,570\end{array}$ & $\begin{array}{r}2,840 \\
2,790 \\
6,595 \\
9,434 \\
21,659\end{array}$ & $\begin{array}{r}2,858 \\
2,740 \\
6,192 \\
9,057 \\
20,847\end{array}$ & $\begin{array}{r}2,677 \\
2,593 \\
5,966 \\
8,573 \\
19,809\end{array}$ & $\begin{array}{r}2,673 \\
2,509 \\
6,106 \\
8,605 \\
19,893\end{array}$ & $\begin{array}{r}2,662 \\
2,530 \\
5,961 \\
8,559 \\
19,712\end{array}$ & $\begin{array}{r}2,609 \\
2,567 \\
5,849 \\
8,336 \\
19,361\end{array}$ & $\begin{array}{r}2,759 \\
2,764 \\
6,362 \\
9,330 \\
21,215\end{array}$ & $\begin{array}{r}2,824 \\
2,817 \\
6,603 \\
9,730 \\
21,974\end{array}$ & $\begin{array}{r}3,023 \\
3,088 \\
7,483 \\
11,586 \\
25,180\end{array}$ & $\begin{array}{r}33,656 \\
33,684 \\
79,289 \\
115,796 \\
262,425\end{array}$ \\
\hline elsewhere & $\begin{array}{l}0-64 \\
65-74 \\
75-84 \\
85+ \\
\text { All ages }\end{array}$ & $\begin{array}{r}12 \\
6 \\
11 \\
9 \\
38\end{array}$ & $\begin{array}{r}11 \\
13 \\
6 \\
6 \\
36\end{array}$ & $\begin{array}{r}9 \\
5 \\
4 \\
2 \\
20\end{array}$ & $\begin{array}{r}13 \\
14 \\
14 \\
6 \\
47\end{array}$ & $\begin{array}{r}15 \\
11 \\
9 \\
2 \\
37\end{array}$ & $\begin{array}{r}19 \\
6 \\
7 \\
3 \\
35\end{array}$ & $\begin{array}{r}19 \\
14 \\
10 \\
2 \\
45\end{array}$ & $\begin{array}{r}21 \\
13 \\
4 \\
4 \\
42\end{array}$ & $\begin{array}{r}18 \\
12 \\
9 \\
5 \\
44\end{array}$ & $\begin{array}{r}11 \\
6 \\
11 \\
1 \\
29\end{array}$ & $\begin{array}{r}11 \\
6 \\
6 \\
3 \\
26\end{array}$ & $\begin{array}{r}15 \\
11 \\
7 \\
8 \\
41\end{array}$ & $\begin{array}{r}174 \\
117 \\
98 \\
51 \\
440\end{array}$ \\
\hline
\end{tabular}


Government Office Regions of England, Wales

\begin{tabular}{|c|c|c|c|c|c|c|c|c|c|c|c|c|c|c|}
\hline & & January & February & March & April & May & June & July & August & September & October & November & December & Total $^{1}$ \\
\hline England, Wales and elsewhere & $\begin{array}{l}0-64 \\
65-74 \\
75-84 \\
85+ \\
\text { All ages }\end{array}$ & $\begin{array}{r}4,930 \\
4,469 \\
7,455 \\
6,103 \\
22,957\end{array}$ & $\begin{array}{r}4,252 \\
3,957 \\
6,721 \\
5,159 \\
20,089\end{array}$ & $\begin{array}{r}4,529 \\
4,150 \\
7,069 \\
5,610 \\
21,358\end{array}$ & $\begin{array}{r}4,341 \\
3,963 \\
6,795 \\
5,413 \\
20,512\end{array}$ & $\begin{array}{r}4,403 \\
3,922 \\
6,298 \\
4,706 \\
19,329\end{array}$ & $\begin{array}{r}4,011 \\
3,662 \\
5,963 \\
4,406 \\
18,042\end{array}$ & $\begin{array}{r}4,269 \\
3,700 \\
6,100 \\
4,560 \\
18,629\end{array}$ & $\begin{array}{r}4,016 \\
3,556 \\
5,848 \\
4,332 \\
17,752\end{array}$ & $\begin{array}{r}4,001 \\
3,621 \\
5,861 \\
4,373 \\
17,856\end{array}$ & $\begin{array}{r}4,254 \\
3,847 \\
6,616 \\
4,955 \\
19,672\end{array}$ & $\begin{array}{r}3,975 \\
4,101 \\
6,599 \\
5,306 \\
19,981\end{array}$ & $\begin{array}{r}4,799 \\
4,690 \\
8,326 \\
6,921 \\
24,736\end{array}$ & $\begin{array}{r}51,780 \\
47,638 \\
79,651 \\
61,844 \\
240,913\end{array}$ \\
\hline North East & $\begin{array}{l}0-64 \\
65-74 \\
75-84 \\
85+ \\
\text { All ages }\end{array}$ & $\begin{array}{r}290 \\
268 \\
418 \\
311 \\
1,287\end{array}$ & $\begin{array}{r}227 \\
227 \\
371 \\
224 \\
1,049\end{array}$ & $\begin{array}{r}237 \\
221 \\
412 \\
242 \\
1,112\end{array}$ & $\begin{array}{r}233 \\
227 \\
363 \\
255 \\
1,078\end{array}$ & $\begin{array}{r}242 \\
253 \\
352 \\
206 \\
1,053\end{array}$ & $\begin{array}{l}226 \\
232 \\
326 \\
179 \\
963\end{array}$ & $\begin{array}{l}223 \\
217 \\
339 \\
217 \\
996\end{array}$ & $\begin{array}{l}209 \\
202 \\
320 \\
182 \\
913\end{array}$ & $\begin{array}{r}243 \\
245 \\
353 \\
188 \\
1,029\end{array}$ & $\begin{array}{r}242 \\
209 \\
371 \\
219 \\
1,041\end{array}$ & $\begin{array}{r}223 \\
249 \\
381 \\
257 \\
1,110\end{array}$ & $\begin{array}{r}238 \\
298 \\
443 \\
307 \\
1,286\end{array}$ & $\begin{array}{r}2,833 \\
2,848 \\
4,449 \\
2,787 \\
12,917\end{array}$ \\
\hline North West & $\begin{array}{l}0-64 \\
65-74 \\
75-84 \\
85+ \\
\text { All ages }\end{array}$ & $\begin{array}{r}741 \\
630 \\
1,094 \\
721 \\
3,186\end{array}$ & $\begin{array}{r}639 \\
611 \\
880 \\
597 \\
2,727\end{array}$ & $\begin{array}{r}652 \\
644 \\
917 \\
671 \\
2,884\end{array}$ & $\begin{array}{r}705 \\
590 \\
866 \\
663 \\
2,824\end{array}$ & $\begin{array}{r}638 \\
600 \\
889 \\
551 \\
2,678\end{array}$ & $\begin{array}{r}619 \\
578 \\
766 \\
496 \\
2,459\end{array}$ & $\begin{array}{r}667 \\
572 \\
849 \\
546 \\
2,634\end{array}$ & $\begin{array}{r}630 \\
518 \\
813 \\
492 \\
2,453\end{array}$ & $\begin{array}{r}574 \\
564 \\
779 \\
474 \\
2,391\end{array}$ & $\begin{array}{r}642 \\
576 \\
904 \\
579 \\
2,701\end{array}$ & $\begin{array}{r}617 \\
646 \\
896 \\
637 \\
2,796\end{array}$ & $\begin{array}{r}728 \\
634 \\
1,133 \\
872 \\
3,367\end{array}$ & $\begin{array}{r}7,852 \\
7,163 \\
10,786 \\
7,299 \\
33,100\end{array}$ \\
\hline Yorkshire and The Humber & $\begin{array}{l}0-64 \\
65-74 \\
75-84 \\
85+ \\
\text { All ages }\end{array}$ & $\begin{array}{r}471 \\
473 \\
781 \\
544 \\
2,269\end{array}$ & $\begin{array}{r}444 \\
418 \\
689 \\
475 \\
2,026\end{array}$ & $\begin{array}{r}500 \\
436 \\
695 \\
511 \\
2,142\end{array}$ & $\begin{array}{r}456 \\
400 \\
711 \\
471 \\
2,038\end{array}$ & $\begin{array}{r}472 \\
412 \\
662 \\
438 \\
1,984\end{array}$ & $\begin{array}{r}434 \\
405 \\
620 \\
452 \\
1,911\end{array}$ & $\begin{array}{r}446 \\
376 \\
594 \\
409 \\
1,825\end{array}$ & $\begin{array}{r}395 \\
355 \\
607 \\
369 \\
1,726\end{array}$ & $\begin{array}{r}421 \\
348 \\
582 \\
405 \\
1,756\end{array}$ & $\begin{array}{r}441 \\
382 \\
647 \\
462 \\
1,932\end{array}$ & $\begin{array}{r}416 \\
413 \\
665 \\
487 \\
1,981\end{array}$ & $\begin{array}{r}509 \\
479 \\
822 \\
637 \\
2,447\end{array}$ & $\begin{array}{r}5,405 \\
4,897 \\
8,075 \\
5,660 \\
24,037\end{array}$ \\
\hline East Midlands & $\begin{array}{l}0-64 \\
65-74 \\
75-84 \\
85+ \\
\text { All ages }\end{array}$ & $\begin{array}{r}385 \\
378 \\
659 \\
470 \\
1,892\end{array}$ & $\begin{array}{r}335 \\
312 \\
567 \\
427 \\
1,641\end{array}$ & $\begin{array}{r}335 \\
356 \\
617 \\
474 \\
1,782\end{array}$ & $\begin{array}{r}365 \\
315 \\
610 \\
469 \\
1,759\end{array}$ & $\begin{array}{r}350 \\
355 \\
544 \\
427 \\
1,676\end{array}$ & $\begin{array}{r}290 \\
302 \\
554 \\
342 \\
1,488\end{array}$ & $\begin{array}{r}375 \\
315 \\
482 \\
384 \\
1,556\end{array}$ & $\begin{array}{r}349 \\
272 \\
511 \\
359 \\
1,491\end{array}$ & $\begin{array}{r}341 \\
299 \\
539 \\
323 \\
1,502\end{array}$ & $\begin{array}{r}368 \\
364 \\
584 \\
387 \\
1,703\end{array}$ & $\begin{array}{r}289 \\
332 \\
573 \\
445 \\
1,639\end{array}$ & $\begin{array}{r}405 \\
454 \\
741 \\
551 \\
2,151\end{array}$ & $\begin{array}{r}4,187 \\
4,054 \\
6,981 \\
5,058 \\
20,280\end{array}$ \\
\hline West Midlands & $\begin{array}{l}0-64 \\
65-74 \\
75-84 \\
85+ \\
\text { All ages }\end{array}$ & $\begin{array}{r}528 \\
503 \\
754 \\
592 \\
2,377\end{array}$ & $\begin{array}{r}434 \\
436 \\
721 \\
527 \\
2,118\end{array}$ & $\begin{array}{r}482 \\
428 \\
738 \\
532 \\
2,180\end{array}$ & $\begin{array}{r}475 \\
430 \\
703 \\
497 \\
2,105\end{array}$ & $\begin{array}{r}471 \\
403 \\
628 \\
453 \\
1,955\end{array}$ & $\begin{array}{r}412 \\
405 \\
610 \\
448 \\
1,875\end{array}$ & $\begin{array}{r}469 \\
407 \\
659 \\
450 \\
1,985\end{array}$ & $\begin{array}{r}412 \\
397 \\
624 \\
459 \\
1,892\end{array}$ & $\begin{array}{r}406 \\
389 \\
612 \\
393 \\
1,800\end{array}$ & $\begin{array}{r}456 \\
434 \\
724 \\
498 \\
2,112\end{array}$ & $\begin{array}{r}415 \\
433 \\
636 \\
527 \\
2,011\end{array}$ & $\begin{array}{r}509 \\
484 \\
929 \\
664 \\
2,586\end{array}$ & $\begin{array}{r}5,469 \\
5,149 \\
8,338 \\
6,040 \\
24,996\end{array}$ \\
\hline East of England & $\begin{array}{l}0-64 \\
65-74 \\
75-84 \\
85+ \\
\text { All ages }\end{array}$ & $\begin{array}{r}476 \\
419 \\
751 \\
663 \\
2,309\end{array}$ & $\begin{array}{r}407 \\
412 \\
748 \\
620 \\
2,187\end{array}$ & $\begin{array}{r}441 \\
387 \\
749 \\
672 \\
2,249\end{array}$ & $\begin{array}{r}401 \\
415 \\
748 \\
590 \\
2,154\end{array}$ & $\begin{array}{r}405 \\
362 \\
673 \\
539 \\
1,979\end{array}$ & $\begin{array}{r}367 \\
333 \\
667 \\
524 \\
1,891\end{array}$ & $\begin{array}{r}342 \\
347 \\
636 \\
511 \\
1,836\end{array}$ & $\begin{array}{r}339 \\
347 \\
568 \\
480 \\
1,734\end{array}$ & $\begin{array}{r}366 \\
371 \\
646 \\
542 \\
1,925\end{array}$ & $\begin{array}{r}377 \\
358 \\
724 \\
599 \\
2,058\end{array}$ & $\begin{array}{r}361 \\
376 \\
670 \\
594 \\
2,001\end{array}$ & $\begin{array}{r}439 \\
440 \\
875 \\
759 \\
2,513\end{array}$ & $\begin{array}{r}4,721 \\
4,567 \\
8,455 \\
7,093 \\
24,836\end{array}$ \\
\hline London & $\begin{array}{l}0-64 \\
65-74 \\
75-84 \\
85+ \\
\text { All ages }\end{array}$ & $\begin{array}{r}584 \\
422 \\
730 \\
601 \\
2,337\end{array}$ & $\begin{array}{r}559 \\
397 \\
661 \\
532 \\
2,149\end{array}$ & $\begin{array}{r}569 \\
436 \\
666 \\
541 \\
2,212\end{array}$ & $\begin{array}{r}518 \\
385 \\
643 \\
570 \\
2,116\end{array}$ & $\begin{array}{r}543 \\
377 \\
569 \\
449 \\
1,938\end{array}$ & $\begin{array}{r}484 \\
352 \\
539 \\
412 \\
1,787\end{array}$ & $\begin{array}{r}515 \\
382 \\
567 \\
430 \\
1,894\end{array}$ & $\begin{array}{r}483 \\
344 \\
508 \\
397 \\
1,732\end{array}$ & $\begin{array}{r}474 \\
339 \\
510 \\
406 \\
1,729\end{array}$ & $\begin{array}{r}488 \\
360 \\
585 \\
506 \\
1,939\end{array}$ & $\begin{array}{r}494 \\
417 \\
663 \\
541 \\
2,115\end{array}$ & $\begin{array}{r}588 \\
479 \\
777 \\
641 \\
2,485\end{array}$ & $\begin{array}{r}6,299 \\
4,690 \\
7,418 \\
6,026 \\
24,433\end{array}$ \\
\hline South East & $\begin{array}{l}0-64 \\
65-74 \\
75-84 \\
85+ \\
\text { All ages }\end{array}$ & $\begin{array}{r}690 \\
657 \\
1,016 \\
1,087 \\
3,450\end{array}$ & $\begin{array}{r}571 \\
481 \\
1,004 \\
811 \\
2,867\end{array}$ & $\begin{array}{r}612 \\
521 \\
1,024 \\
899 \\
3,056\end{array}$ & $\begin{array}{r}576 \\
552 \\
1,008 \\
916 \\
3,052\end{array}$ & $\begin{array}{r}574 \\
502 \\
923 \\
782 \\
2,781\end{array}$ & $\begin{array}{r}547 \\
471 \\
862 \\
740 \\
2,620\end{array}$ & $\begin{array}{r}539 \\
474 \\
889 \\
772 \\
2,674\end{array}$ & $\begin{array}{r}558 \\
503 \\
880 \\
782 \\
2,723\end{array}$ & $\begin{array}{r}546 \\
429 \\
818 \\
776 \\
2,569\end{array}$ & $\begin{array}{r}580 \\
517 \\
935 \\
825 \\
2,857\end{array}$ & $\begin{array}{r}534 \\
575 \\
916 \\
868 \\
2,893\end{array}$ & $\begin{array}{r}659 \\
667 \\
1,236 \\
1,252 \\
3,814\end{array}$ & $\begin{array}{r}6,986 \\
6,349 \\
11,511 \\
10,510 \\
35,356\end{array}$ \\
\hline South West & $\begin{array}{l}0-64 \\
65-74 \\
75-84 \\
85+ \\
\text { All ages }\end{array}$ & $\begin{array}{r}434 \\
436 \\
776 \\
759 \\
2,405\end{array}$ & $\begin{array}{r}365 \\
360 \\
650 \\
631 \\
2,006\end{array}$ & $\begin{array}{r}413 \\
422 \\
747 \\
702 \\
2,284\end{array}$ & $\begin{array}{r}337 \\
373 \\
699 \\
660 \\
2,069\end{array}$ & $\begin{array}{r}391 \\
350 \\
652 \\
571 \\
1,964\end{array}$ & $\begin{array}{r}378 \\
345 \\
633 \\
551 \\
1,907\end{array}$ & $\begin{array}{r}377 \\
348 \\
655 \\
552 \\
1,932\end{array}$ & $\begin{array}{r}343 \\
356 \\
631 \\
530 \\
1,860\end{array}$ & $\begin{array}{r}354 \\
376 \\
662 \\
601 \\
1,993\end{array}$ & $\begin{array}{r}356 \\
382 \\
697 \\
565 \\
2,000\end{array}$ & $\begin{array}{r}350 \\
367 \\
715 \\
640 \\
2,072\end{array}$ & $\begin{array}{r}419 \\
438 \\
823 \\
839 \\
2,519\end{array}$ & $\begin{array}{r}4,517 \\
4,553 \\
8,340 \\
7,601 \\
25,011\end{array}$ \\
\hline Wales & $\begin{array}{l}0-64 \\
65-74 \\
75-84 \\
85+ \\
\text { All ages }\end{array}$ & $\begin{array}{r}298 \\
272 \\
461 \\
350 \\
1,381\end{array}$ & $\begin{array}{r}236 \\
290 \\
421 \\
312 \\
1,259\end{array}$ & $\begin{array}{r}255 \\
289 \\
498 \\
362 \\
1,404\end{array}$ & $\begin{array}{r}245 \\
264 \\
438 \\
320 \\
1,267\end{array}$ & $\begin{array}{r}285 \\
297 \\
398 \\
286 \\
1,266\end{array}$ & $\begin{array}{r}214 \\
223 \\
374 \\
257 \\
1,068\end{array}$ & $\begin{array}{r}283 \\
246 \\
417 \\
283 \\
1,229\end{array}$ & $\begin{array}{r}246 \\
251 \\
376 \\
279 \\
1,152\end{array}$ & $\begin{array}{r}238 \\
244 \\
351 \\
259 \\
1,092\end{array}$ & $\begin{array}{r}263 \\
254 \\
430 \\
311 \\
1,258\end{array}$ & $\begin{array}{r}244 \\
280 \\
475 \\
307 \\
1,306\end{array}$ & $\begin{array}{r}278 \\
300 \\
541 \\
397 \\
1,516\end{array}$ & $\begin{array}{r}3,085 \\
3,210 \\
5,180 \\
3,723 \\
15,198\end{array}$ \\
\hline England & $\begin{array}{l}0-64 \\
65-74 \\
75-84 \\
85+ \\
\text { All ages }\end{array}$ & $\begin{array}{r}4,599 \\
4,186 \\
6,979 \\
5,748 \\
21,512\end{array}$ & $\begin{array}{r}3,981 \\
3,654 \\
6,291 \\
4,844 \\
18,770\end{array}$ & $\begin{array}{r}4,241 \\
3,851 \\
6,565 \\
5,244 \\
19,901\end{array}$ & $\begin{array}{r}4,066 \\
3,687 \\
6,351 \\
5,091 \\
19,195\end{array}$ & $\begin{array}{r}4,086 \\
3,614 \\
5,892 \\
4,416 \\
18,008\end{array}$ & $\begin{array}{r}3,757 \\
3,423 \\
5,577 \\
4,144 \\
16,901\end{array}$ & $\begin{array}{r}3,953 \\
3,438 \\
5,670 \\
4,271 \\
17,332\end{array}$ & $\begin{array}{r}3,718 \\
3,294 \\
5,462 \\
4,050 \\
16,524\end{array}$ & $\begin{array}{r}3,725 \\
3,360 \\
5,501 \\
4,108 \\
16,694\end{array}$ & $\begin{array}{r}3,950 \\
3,582 \\
6,171 \\
4,640 \\
18,343\end{array}$ & $\begin{array}{r}3,699 \\
3,808 \\
6,115 \\
4,996 \\
18,618\end{array}$ & $\begin{array}{r}4,494 \\
4,373 \\
7,779 \\
6,522 \\
23,168\end{array}$ & $\begin{array}{r}48,269 \\
44,270 \\
74,353 \\
58,074 \\
224,966\end{array}$ \\
\hline England and Wales & $\begin{array}{l}0-64 \\
65-74 \\
75-84 \\
85+ \\
\text { All ages }\end{array}$ & $\begin{array}{r}4,897 \\
4,458 \\
7,440 \\
6,098 \\
22,893\end{array}$ & $\begin{array}{r}4,217 \\
3,944 \\
6,712 \\
5,156 \\
20,029\end{array}$ & $\begin{array}{r}4,496 \\
4,140 \\
7,063 \\
5,606 \\
21,305\end{array}$ & $\begin{array}{r}4,311 \\
3,951 \\
6,789 \\
5,411 \\
20,462\end{array}$ & $\begin{array}{r}4,371 \\
3,911 \\
6,290 \\
4,702 \\
19,274\end{array}$ & $\begin{array}{r}3,971 \\
3,646 \\
5,951 \\
4,401 \\
17,969\end{array}$ & $\begin{array}{r}4,236 \\
3,684 \\
6,087 \\
4,554 \\
18,561\end{array}$ & $\begin{array}{r}3,964 \\
3,545 \\
5,838 \\
4,329 \\
17,676\end{array}$ & $\begin{array}{r}3,963 \\
3,604 \\
5,852 \\
4,367 \\
17,786\end{array}$ & $\begin{array}{r}4,213 \\
3,836 \\
6,601 \\
4,951 \\
19,601\end{array}$ & $\begin{array}{r}3,943 \\
4,088 \\
6,590 \\
5,303 \\
19,924\end{array}$ & $\begin{array}{r}4,772 \\
4,673 \\
8,320 \\
6,919 \\
24,684\end{array}$ & $\begin{array}{r}51,354 \\
47,480 \\
79,533 \\
61,797 \\
240,164\end{array}$ \\
\hline elsewhere & $\begin{array}{l}0-64 \\
65-74 \\
75-84 \\
85+ \\
\text { All ages }\end{array}$ & $\begin{array}{r}33 \\
11 \\
15 \\
5 \\
64\end{array}$ & $\begin{array}{r}35 \\
13 \\
9 \\
3 \\
60\end{array}$ & $\begin{array}{r}33 \\
10 \\
6 \\
4 \\
53\end{array}$ & $\begin{array}{r}30 \\
12 \\
6 \\
2 \\
50\end{array}$ & $\begin{array}{r}32 \\
11 \\
8 \\
4 \\
55\end{array}$ & $\begin{array}{r}40 \\
16 \\
12 \\
5 \\
73\end{array}$ & $\begin{array}{r}33 \\
16 \\
13 \\
6 \\
68\end{array}$ & $\begin{array}{r}52 \\
11 \\
10 \\
3 \\
76\end{array}$ & $\begin{array}{r}38 \\
17 \\
9 \\
6 \\
70\end{array}$ & $\begin{array}{r}41 \\
11 \\
15 \\
4 \\
71\end{array}$ & $\begin{array}{r}32 \\
13 \\
9 \\
3 \\
57\end{array}$ & $\begin{array}{r}27 \\
17 \\
6 \\
2 \\
52\end{array}$ & $\begin{array}{r}426 \\
158 \\
118 \\
47 \\
749\end{array}$ \\
\hline
\end{tabular}


Government Office Regions of England, Wales

\begin{tabular}{|c|c|c|c|c|c|c|c|c|c|c|c|c|c|c|}
\hline & & January & February & March & April & May & June & July & August & September & October & November & December & Total $^{1}$ \\
\hline England, Wales and elsewhere & $\begin{array}{l}0-64 \\
65-74 \\
75-84 \\
85+ \\
\text { All ages }\end{array}$ & $\begin{array}{r}3,060 \\
3,196 \\
7,616 \\
12,038 \\
25,910\end{array}$ & $\begin{array}{r}2,800 \\
2,716 \\
6,581 \\
10,002 \\
22,099\end{array}$ & $\begin{array}{r}2,945 \\
2,933 \\
6,935 \\
10,580 \\
23,393\end{array}$ & $\begin{array}{r}2,864 \\
2,809 \\
6,763 \\
10,299 \\
22,735\end{array}$ & $\begin{array}{r}2,812 \\
2,735 \\
6,032 \\
9,406 \\
20,985\end{array}$ & $\begin{array}{r}2,562 \\
2,547 \\
5,680 \\
8,417 \\
19,206\end{array}$ & $\begin{array}{r}2,732 \\
2,632 \\
5,848 \\
8,505 \\
19,717\end{array}$ & $\begin{array}{r}2,664 \\
2,539 \\
5,607 \\
8,426 \\
19,236\end{array}$ & $\begin{array}{r}2,653 \\
2,515 \\
5,506 \\
8,429 \\
19,103\end{array}$ & $\begin{array}{r}2,876 \\
2,803 \\
6,125 \\
9,474 \\
21,278\end{array}$ & $\begin{array}{r}2,757 \\
2,771 \\
6,452 \\
10,015 \\
21,995\end{array}$ & $\begin{array}{r}3,216 \\
3,601 \\
8,341 \\
14,012 \\
29,170\end{array}$ & $\begin{array}{r}33,941 \\
33,797 \\
77,486 \\
119,603 \\
264,827\end{array}$ \\
\hline North East & $\begin{array}{l}0-64 \\
65-74 \\
75-84 \\
85+ \\
\text { All ages }\end{array}$ & $\begin{array}{r}191 \\
208 \\
493 \\
604 \\
1,496\end{array}$ & $\begin{array}{r}160 \\
161 \\
402 \\
501 \\
1,224\end{array}$ & $\begin{array}{r}165 \\
173 \\
390 \\
495 \\
1,223\end{array}$ & $\begin{array}{r}145 \\
173 \\
416 \\
464 \\
1,198\end{array}$ & $\begin{array}{r}148 \\
166 \\
353 \\
427 \\
1,094\end{array}$ & $\begin{array}{l}117 \\
158 \\
329 \\
367 \\
971\end{array}$ & $\begin{array}{r}158 \\
145 \\
348 \\
381 \\
1,032\end{array}$ & $\begin{array}{r}141 \\
131 \\
348 \\
387 \\
1,007\end{array}$ & $\begin{array}{r}136 \\
153 \\
328 \\
418 \\
1,035\end{array}$ & $\begin{array}{r}166 \\
161 \\
353 \\
442 \\
1,122\end{array}$ & $\begin{array}{r}159 \\
175 \\
385 \\
444 \\
1,163\end{array}$ & $\begin{array}{r}176 \\
203 \\
513 \\
667 \\
1,559\end{array}$ & $\begin{array}{r}1,862 \\
2,007 \\
4,658 \\
5,597 \\
14,124\end{array}$ \\
\hline North West & $\begin{array}{l}0-64 \\
65-74 \\
75-84 \\
85+ \\
\text { All ages }\end{array}$ & $\begin{array}{r}426 \\
516 \\
1,051 \\
1,573 \\
3,566\end{array}$ & $\begin{array}{r}413 \\
441 \\
947 \\
1,334 \\
3,135\end{array}$ & $\begin{array}{r}451 \\
445 \\
1,025 \\
1,452 \\
3,373\end{array}$ & $\begin{array}{r}440 \\
434 \\
993 \\
1,383 \\
3,250\end{array}$ & $\begin{array}{r}420 \\
418 \\
880 \\
1,190 \\
2,908\end{array}$ & $\begin{array}{r}395 \\
387 \\
794 \\
1,086 \\
2,662\end{array}$ & $\begin{array}{r}420 \\
428 \\
828 \\
1,098 \\
2,774\end{array}$ & $\begin{array}{r}389 \\
419 \\
795 \\
1,110 \\
2,713\end{array}$ & $\begin{array}{r}401 \\
379 \\
762 \\
1,041 \\
2,583\end{array}$ & $\begin{array}{r}400 \\
411 \\
891 \\
1,176 \\
2,878\end{array}$ & $\begin{array}{r}408 \\
457 \\
955 \\
1,348 \\
3,168\end{array}$ & $\begin{array}{r}502 \\
607 \\
1,218 \\
1,822 \\
4,149\end{array}$ & $\begin{array}{r}5,065 \\
5,342 \\
11,139 \\
15,613 \\
37,159\end{array}$ \\
\hline Yorkshire and The Humber & $\begin{array}{l}0-64 \\
65-74 \\
75-84 \\
85+ \\
\text { All ages }\end{array}$ & $\begin{array}{r}303 \\
337 \\
811 \\
1,124 \\
2,575\end{array}$ & $\begin{array}{r}281 \\
251 \\
666 \\
949 \\
2,147\end{array}$ & $\begin{array}{r}300 \\
312 \\
683 \\
1,007 \\
2,302\end{array}$ & $\begin{array}{r}269 \\
288 \\
672 \\
1,014 \\
2,243\end{array}$ & $\begin{array}{r}269 \\
299 \\
647 \\
884 \\
2,099\end{array}$ & $\begin{array}{r}270 \\
294 \\
594 \\
781 \\
1,939\end{array}$ & $\begin{array}{r}301 \\
268 \\
623 \\
793 \\
1,985\end{array}$ & $\begin{array}{r}294 \\
264 \\
543 \\
778 \\
1,879\end{array}$ & $\begin{array}{r}273 \\
263 \\
551 \\
808 \\
1,895\end{array}$ & $\begin{array}{r}290 \\
296 \\
603 \\
888 \\
2,077\end{array}$ & $\begin{array}{r}292 \\
301 \\
691 \\
939 \\
2,223\end{array}$ & $\begin{array}{r}310 \\
397 \\
837 \\
1,318 \\
2,862 \\
\end{array}$ & $\begin{array}{r}3,452 \\
3,570 \\
7,921 \\
11,283 \\
26,226\end{array}$ \\
\hline East Midlands & $\begin{array}{l}0-64 \\
65-74 \\
75-84 \\
85+ \\
\text { All ages }\end{array}$ & $\begin{array}{r}263 \\
240 \\
630 \\
971 \\
2,104\end{array}$ & $\begin{array}{r}231 \\
213 \\
559 \\
770 \\
1,773\end{array}$ & $\begin{array}{r}250 \\
254 \\
555 \\
808 \\
1,867\end{array}$ & $\begin{array}{r}222 \\
243 \\
565 \\
856 \\
1,886\end{array}$ & $\begin{array}{r}217 \\
222 \\
483 \\
729 \\
1,651\end{array}$ & $\begin{array}{r}227 \\
211 \\
488 \\
696 \\
1,622\end{array}$ & $\begin{array}{r}259 \\
215 \\
484 \\
704 \\
1,662\end{array}$ & $\begin{array}{r}209 \\
180 \\
480 \\
693 \\
1,562\end{array}$ & $\begin{array}{r}220 \\
222 \\
461 \\
657 \\
1,560\end{array}$ & $\begin{array}{r}228 \\
219 \\
521 \\
756 \\
1,724\end{array}$ & $\begin{array}{r}230 \\
248 \\
571 \\
824 \\
1,873\end{array}$ & $\begin{array}{r}283 \\
277 \\
719 \\
1,154 \\
2,433\end{array}$ & $\begin{array}{r}2,839 \\
2,744 \\
6,516 \\
9,618 \\
21,717\end{array}$ \\
\hline West Midlands & $\begin{array}{l}0-64 \\
65-74 \\
75-84 \\
85+ \\
\text { All ages }\end{array}$ & $\begin{array}{r}324 \\
351 \\
793 \\
1,241 \\
2,709\end{array}$ & $\begin{array}{r}309 \\
280 \\
707 \\
927 \\
2,223\end{array}$ & $\begin{array}{r}333 \\
333 \\
724 \\
998 \\
2,388\end{array}$ & $\begin{array}{r}321 \\
296 \\
685 \\
1,010 \\
2,312\end{array}$ & $\begin{array}{r}276 \\
316 \\
646 \\
919 \\
2,157\end{array}$ & $\begin{array}{r}264 \\
234 \\
617 \\
821 \\
1,936\end{array}$ & $\begin{array}{r}282 \\
272 \\
609 \\
877 \\
2,040\end{array}$ & $\begin{array}{r}282 \\
254 \\
544 \\
832 \\
1,912\end{array}$ & $\begin{array}{r}263 \\
285 \\
590 \\
822 \\
1,960\end{array}$ & $\begin{array}{r}291 \\
285 \\
634 \\
908 \\
2,118\end{array}$ & $\begin{array}{r}274 \\
282 \\
623 \\
975 \\
2,154\end{array}$ & $\begin{array}{r}323 \\
380 \\
873 \\
1,395 \\
2,971\end{array}$ & $\begin{array}{r}3,542 \\
3,568 \\
8,045 \\
11,725 \\
26,880\end{array}$ \\
\hline East of England & $\begin{array}{l}0-64 \\
65-74 \\
75-84 \\
85+ \\
\text { All ages }\end{array}$ & $\begin{array}{r}301 \\
300 \\
756 \\
1,289 \\
2,646\end{array}$ & $\begin{array}{r}254 \\
254 \\
656 \\
1,103 \\
2,267\end{array}$ & $\begin{array}{r}282 \\
288 \\
737 \\
1,172 \\
2,479\end{array}$ & $\begin{array}{r}298 \\
282 \\
704 \\
1,082 \\
2,366\end{array}$ & $\begin{array}{r}305 \\
270 \\
588 \\
1,025 \\
2,188\end{array}$ & $\begin{array}{r}215 \\
251 \\
562 \\
927 \\
1,955\end{array}$ & $\begin{array}{r}215 \\
280 \\
600 \\
929 \\
2,024\end{array}$ & $\begin{array}{r}288 \\
284 \\
557 \\
959 \\
2,088\end{array}$ & $\begin{array}{r}241 \\
224 \\
559 \\
913 \\
1,937\end{array}$ & $\begin{array}{r}277 \\
304 \\
607 \\
1,087 \\
2,275\end{array}$ & $\begin{array}{r}269 \\
238 \\
706 \\
1,106 \\
2,319\end{array}$ & $\begin{array}{r}322 \\
320 \\
838 \\
1,494 \\
2,974\end{array}$ & $\begin{array}{r}3,267 \\
3,295 \\
7,870 \\
13,086 \\
27,518\end{array}$ \\
\hline London & $\begin{array}{l}0-64 \\
65-74 \\
75-84 \\
85+ \\
\text { All ages }\end{array}$ & $\begin{array}{r}355 \\
311 \\
719 \\
1,079 \\
2,464\end{array}$ & $\begin{array}{r}345 \\
289 \\
624 \\
939 \\
2,197\end{array}$ & $\begin{array}{r}344 \\
282 \\
709 \\
1,003 \\
2,338\end{array}$ & $\begin{array}{r}321 \\
274 \\
623 \\
960 \\
2,178\end{array}$ & $\begin{array}{r}335 \\
283 \\
546 \\
883 \\
2,047\end{array}$ & $\begin{array}{r}307 \\
243 \\
533 \\
791 \\
1,874\end{array}$ & $\begin{array}{r}292 \\
243 \\
569 \\
788 \\
1,892\end{array}$ & $\begin{array}{r}296 \\
263 \\
514 \\
764 \\
1,837\end{array}$ & $\begin{array}{r}303 \\
217 \\
458 \\
772 \\
1,750\end{array}$ & $\begin{array}{r}321 \\
275 \\
582 \\
898 \\
2,076\end{array}$ & $\begin{array}{r}338 \\
263 \\
584 \\
913 \\
2,098\end{array}$ & $\begin{array}{r}386 \\
346 \\
750 \\
1,325 \\
2,807\end{array}$ & $\begin{array}{r}3,943 \\
3,289 \\
7,211 \\
11,115 \\
25,558\end{array}$ \\
\hline South East & $\begin{array}{l}0-64 \\
65-74 \\
75-84 \\
85+ \\
\text { All ages }\end{array}$ & $\begin{array}{r}397 \\
405 \\
1,070 \\
1,950 \\
3,822\end{array}$ & $\begin{array}{r}392 \\
398 \\
929 \\
1,684 \\
3,403\end{array}$ & $\begin{array}{r}364 \\
390 \\
992 \\
1,804 \\
3,550\end{array}$ & $\begin{array}{r}387 \\
387 \\
1,005 \\
1,692 \\
3,471\end{array}$ & $\begin{array}{r}393 \\
355 \\
876 \\
1,596 \\
3,220\end{array}$ & $\begin{array}{r}378 \\
387 \\
829 \\
1,446 \\
3,040\end{array}$ & $\begin{array}{r}372 \\
358 \\
826 \\
1,384 \\
2,940\end{array}$ & $\begin{array}{r}363 \\
358 \\
820 \\
1,412 \\
2,953\end{array}$ & $\begin{array}{r}340 \\
347 \\
858 \\
1,462 \\
3,007\end{array}$ & $\begin{array}{r}412 \\
389 \\
918 \\
1,616 \\
3,335\end{array}$ & $\begin{array}{r}369 \\
371 \\
933 \\
1,644 \\
3,317\end{array}$ & $\begin{array}{r}419 \\
466 \\
1,210 \\
2,374 \\
4,469\end{array}$ & $\begin{array}{r}4,586 \\
4,611 \\
11,266 \\
20,064 \\
40,527\end{array}$ \\
\hline South West & $\begin{array}{l}0-64 \\
65-74 \\
75-84 \\
85+ \\
\text { All ages }\end{array}$ & $\begin{array}{r}296 \\
337 \\
815 \\
1,463 \\
2,911\end{array}$ & $\begin{array}{r}231 \\
226 \\
658 \\
1,175 \\
2,290\end{array}$ & $\begin{array}{r}266 \\
262 \\
652 \\
1,177 \\
2,357\end{array}$ & $\begin{array}{r}251 \\
241 \\
665 \\
1,204 \\
2,361\end{array}$ & $\begin{array}{r}238 \\
230 \\
625 \\
1,143 \\
2,236\end{array}$ & $\begin{array}{r}219 \\
211 \\
573 \\
990 \\
1,993\end{array}$ & $\begin{array}{r}245 \\
237 \\
600 \\
985 \\
2,067\end{array}$ & $\begin{array}{r}230 \\
220 \\
651 \\
988 \\
2,089\end{array}$ & $\begin{array}{r}264 \\
232 \\
597 \\
1,026 \\
2,119\end{array}$ & $\begin{array}{r}271 \\
246 \\
623 \\
1,124 \\
2,264\end{array}$ & $\begin{array}{r}239 \\
243 \\
611 \\
1,245 \\
2,338\end{array}$ & $\begin{array}{r}260 \\
323 \\
845 \\
1,637 \\
3,065\end{array}$ & $\begin{array}{r}3,010 \\
3,008 \\
7,915 \\
14,157 \\
28,090\end{array}$ \\
\hline Wales & $\begin{array}{l}0-64 \\
65-74 \\
75-84 \\
85+ \\
\text { All ages }\end{array}$ & $\begin{array}{r}193 \\
180 \\
472 \\
734 \\
1,579\end{array}$ & $\begin{array}{r}170 \\
197 \\
427 \\
618 \\
1,412\end{array}$ & $\begin{array}{r}175 \\
186 \\
464 \\
661 \\
1,486\end{array}$ & $\begin{array}{r}190 \\
182 \\
429 \\
632 \\
1,433\end{array}$ & $\begin{array}{r}183 \\
171 \\
379 \\
606 \\
1,339\end{array}$ & $\begin{array}{r}156 \\
163 \\
358 \\
503 \\
1,180\end{array}$ & $\begin{array}{r}171 \\
173 \\
351 \\
556 \\
1,251\end{array}$ & $\begin{array}{r}155 \\
158 \\
343 \\
501 \\
1,157\end{array}$ & $\begin{array}{r}190 \\
185 \\
334 \\
497 \\
1,206\end{array}$ & $\begin{array}{r}200 \\
207 \\
391 \\
568 \\
1,366\end{array}$ & $\begin{array}{r}171 \\
184 \\
387 \\
571 \\
1,313\end{array}$ & $\begin{array}{r}220 \\
272 \\
526 \\
816 \\
1,834\end{array}$ & $\begin{array}{r}2,174 \\
2,258 \\
4,861 \\
7,263 \\
16,556\end{array}$ \\
\hline England & $\begin{array}{l}0-64 \\
65-74 \\
75-84 \\
85+ \\
\text { All ages }\end{array}$ & $\begin{array}{r}2,856 \\
3,005 \\
7,138 \\
11,294 \\
24,293\end{array}$ & $\begin{array}{r}2,616 \\
2,513 \\
6,148 \\
9,382 \\
20,659\end{array}$ & $\begin{array}{r}2,755 \\
2,739 \\
6,467 \\
9,916 \\
21,877\end{array}$ & $\begin{array}{r}2,654 \\
2,618 \\
6,328 \\
9,665 \\
21,265\end{array}$ & $\begin{array}{r}2,601 \\
2,559 \\
5,644 \\
8,796 \\
19,600\end{array}$ & $\begin{array}{r}2,392 \\
2,376 \\
5,319 \\
7,905 \\
17,992\end{array}$ & $\begin{array}{r}2,544 \\
2,446 \\
5,487 \\
7,939 \\
18,416\end{array}$ & $\begin{array}{r}2,492 \\
2,373 \\
5,252 \\
7,923 \\
18,040\end{array}$ & $\begin{array}{r}2,441 \\
2,322 \\
5,164 \\
7,919 \\
17,846\end{array}$ & $\begin{array}{r}2,656 \\
2,586 \\
5,732 \\
8,895 \\
19,869\end{array}$ & $\begin{array}{r}2,578 \\
2,578 \\
6,059 \\
9,438 \\
20,653\end{array}$ & $\begin{array}{r}2,981 \\
3,319 \\
7,803 \\
13,186 \\
27,289\end{array}$ & $\begin{array}{r}31,566 \\
31,434 \\
72,541 \\
112,258 \\
247,799\end{array}$ \\
\hline England and Wales & $\begin{array}{l}0-64 \\
65-74 \\
75-84 \\
85+ \\
\text { All ages }\end{array}$ & $\begin{array}{r}3,049 \\
3,185 \\
7,610 \\
12,028 \\
25,872\end{array}$ & $\begin{array}{r}2,786 \\
2,710 \\
6,575 \\
10,000 \\
22,071\end{array}$ & $\begin{array}{r}2,930 \\
2,925 \\
6,931 \\
10,577 \\
23,363\end{array}$ & $\begin{array}{r}2,844 \\
2,800 \\
6,757 \\
10,297 \\
22,698\end{array}$ & $\begin{array}{r}2,784 \\
2,730 \\
6,023 \\
9,402 \\
20,939\end{array}$ & $\begin{array}{r}2,548 \\
2,539 \\
5,677 \\
8,408 \\
19,172\end{array}$ & $\begin{array}{r}2,715 \\
2,619 \\
5,838 \\
8,495 \\
19,667\end{array}$ & $\begin{array}{r}2,647 \\
2,531 \\
5,595 \\
8,424 \\
19,197\end{array}$ & $\begin{array}{r}2,631 \\
2,507 \\
5,498 \\
8,416 \\
19,052\end{array}$ & $\begin{array}{r}2,856 \\
2,793 \\
6,123 \\
9,463 \\
21,235\end{array}$ & $\begin{array}{r}2,749 \\
2,762 \\
6,446 \\
10,009 \\
21,966\end{array}$ & $\begin{array}{r}3,201 \\
3,591 \\
8,329 \\
14,002 \\
29,123\end{array}$ & $\begin{array}{r}33,740 \\
33,692 \\
77,402 \\
119,521 \\
264,355\end{array}$ \\
\hline elsewhere & $\begin{array}{l}0-64 \\
65-74 \\
75-84 \\
85+ \\
\text { All ages }\end{array}$ & $\begin{array}{r}11 \\
11 \\
6 \\
10 \\
38\end{array}$ & $\begin{array}{r}14 \\
6 \\
6 \\
2 \\
28\end{array}$ & $\begin{array}{r}15 \\
8 \\
4 \\
3 \\
30\end{array}$ & $\begin{array}{r}20 \\
9 \\
6 \\
2 \\
37\end{array}$ & $\begin{array}{r}28 \\
5 \\
9 \\
4 \\
46\end{array}$ & $\begin{array}{r}14 \\
8 \\
3 \\
9 \\
34\end{array}$ & $\begin{array}{l}17 \\
13 \\
10 \\
10 \\
50\end{array}$ & $\begin{array}{r}17 \\
8 \\
12 \\
2 \\
39\end{array}$ & $\begin{array}{r}22 \\
8 \\
8 \\
13 \\
51\end{array}$ & $\begin{array}{r}20 \\
10 \\
2 \\
11 \\
43\end{array}$ & $\begin{array}{r}8 \\
9 \\
6 \\
6 \\
29\end{array}$ & $\begin{array}{l}15 \\
10 \\
12 \\
10 \\
47\end{array}$ & $\begin{array}{r}201 \\
105 \\
84 \\
82 \\
472\end{array}$ \\
\hline
\end{tabular}

